\title{
Progress of the injection laser system of SG-II
}

Wei Fan, Youen Jiang, Jiangfeng Wang, Xiaochao Wang, Dajie Huang, Xinghua Lu, Hui Wei,

Guoyang Li, Xue Pan, Zhi Qiao, Chao Wang, He Cheng, Peng Zhang, Wenfa Huang, Zhuli Xiao,

Shengjia Zhang, Xuechun Li, Jianqiang Zhu, and Zunqi Lin

National Laboratory on High Power Laser and Physics, Shanghai Institute of Optics and Fine Mechanics, Chinese Academy of Sciences, Shanghai 201800, China

(Received 21 November 2017; revised 26 January 2018; accepted 16 March 2018)

\begin{abstract}
A high power laser system was used to drive the ignition of inertial confinement fusion (ICF), of which the high energy, the uniform focal spot, the accurate laser waveform, and the synchronization between the laser beams are key parameters. To accomplish this, global laser characteristics control should be assured, which was the main purpose of the injection laser system. In this paper, the key technological progress involved in the improvement of the performance of the injection laser of SG-II is reported, including frequency domain control, time domain control, near-field spatial shaping, preamplifier technology, and the optical parametric chirped pulse amplification pump source.
\end{abstract}

Keywords: amplifier; injection laser; pulse shaping; spatial shaping; synchronization

\section{Introduction}

Inertial confinement fusion (ICF) is thought to be a promising approach to produce clean energy. The utilization of a solid-state high power laser driver is one of the key elements involved in achieving $\mathrm{ICF}^{[1]}$. The key requirements for the high power laser driver are high energy, uniform focal spot, agile temporal pulse shape, time synchronization control, and beam target coupling. Achievement of these requirements is based on a very complex facility. As a result, all ICF laser drivers include a front end, pre-amplifier, main amplifier, transport, optics assembly (for frequency conversion, and focusing on the target chamber center), etc., as shown in Figure $1^{[1-5]}$. The SG-II facility includes two bundles of eight beams and one 9th beam. The 9th beam can provide a variety of injection methods, laser wavelength $(1 \omega, 2 \omega, 3 \omega)$, and pulse widths, as well as the experimental parameters for different forms of focal distribution. Thus, good backlight or probe experimental conditions are achieved ${ }^{[6-8]}$. SG-II is a multi-function laser system platform, which has a proven track record of use for ICF and other physical research.

The front end of the SG-II has four functions ${ }^{[1,9-11]}$.

(1) To provide different kinds of seed sources for the whole laser system.

(2) To provide globe control ability for the laser pulse, such as the control of the temporal pulse shape, and the

Correspondence to: W. Fan, No. 390, Qinghe Road, Jiading, Shanghai 201800, China. Email: fanweil@siom.ac.cn spectrum characteristics, the spatial intensity shape, and the synchronization between the different kinds of the laser beams.

(3) To provide the monitor and feedback of safe control of the entire laser system to ensure the protection of the large aperture optical components and the effectiveness of the physical experiments.

(4) To provide different kinds of light timescale, electric timescale or other trigger signals required for physical measurements.

The pre-amplifier has three main functions.

(1) To amplify the laser to joule magnitude.

(2) To control the near-field intensity distribution.

(3) To provide the laser source for beam alignment.

All the technologies of the injection laser system including the front end and pre-amplifier are implemented to facilitate convenient and effective globe control of the laser characters ${ }^{[1-5,9-11]}$, including frequency domain, time domain, spatial domain, etc.

\section{The injection laser system of SG-II}

\subsection{The first generation of the injection laser system of $S G$}

During the early period of construction of SG high power laser facility, the front end was based on a solid-state laser operating in the time domain, that is, the shaped laser pulse 


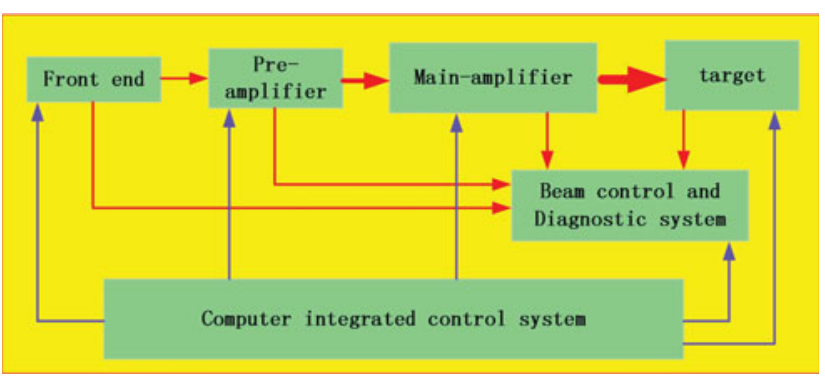

Figure 1. High power laser diagram.

had a smooth time envelope and there was synchronization between different laser beams.

In the 1990s, laser diode (LD) pumped single-longitudinalmode lasers and laser trigger synchronization were adopted by the SG facility. Four key technologies were required, which presented significant challenges during implementation and included single-longitudinal seed lasers, laser pulse waveform shaping technology, short pulse seed lasers, and synchronization between long nanosecond shaped lasers and short pulse lasers. These challenges were eventually effectively addressed.

The primary function of the seed laser source is to acquire a smooth time envelope to avoid damage to the optical mirrors caused by abnormally high peak laser outputs. The physical and technological challenges associated with cavity length control of the $Q$-switched single-mode oscillator were solved, and the laser can operate with a stable single mode for a long time period ${ }^{[12]}$. The shaped electric pulse, which was produced by a high-voltage electrical pulse transmitted through the micro-strip line, was used to trigger a Pockels electrical switch to achieve a shaped laser pulse ${ }^{[13]}$.

Short pulse laser output was realized using a Nd:YAG passive mode locking technique ${ }^{[14]}$. Based on the characteristics of the GaAs photoconductive switching resistors with a linear increase in the intensity of illumination, high sensitivity and ultrafast laser positive feedback loops were developed for the first time. On this basis, the mode locking of the Nd:YAG laser, automatic tracking of the selection of single-longitudinal modes and $Q$ switching were realized. The synchronization accuracy between the nanosecond laser and the picosecond laser is in the range of nanosecond orders of magnitude ${ }^{[14-16]}$.

The above work played a key role in the successful development of SG-I and SG-II devices.

\subsection{The second generation of the injection laser system}

With the development of communication technology, the integrated waveguide front-end system was developed for the first time in China ${ }^{[11,17]}$. The SG-II series of new front-end prototypes, with the integration of a single-longitudinal fiber laser ${ }^{[18,19]}$, fiber amplifier, high-speed integrated waveguide modulator ${ }^{[20]}$, etc., have been featured in the SG-II since 2004 as shown in Figure 2. The specification and the performance standards fully met the engineering design needs of the SG-II. Using short laser pulse trigger in combination with silicon photoconductive switch technology, a high-precision synchronous output was achieved ${ }^{[21]}$.

In recent years, with the increasing demand for physical experiments and the development of unit and integrated technology, high-precision control of synchronization, spectrum, time-power curve, polarization, near field, and energy stability, the function and scale of injection laser systems have expanded.

\section{The functions of injection laser system of SG-II}

The current SG-II series includes two bundles of eight beams of long nanosecond shaped pulses in addition to a 9th beam. The 9th beam is a diagnostic laser pulse, which can output either a long nanosecond shaped laser pulse or a short picosecond pulse as a high-energy PW laser or a diagnostics laser.

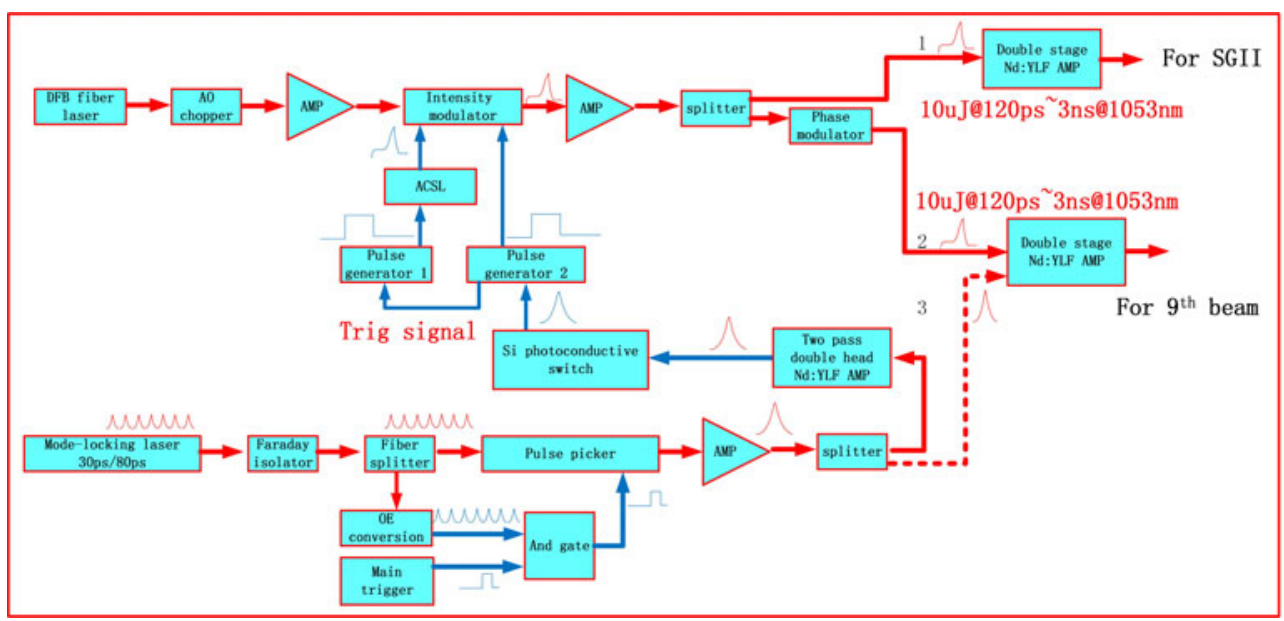

Figure 2. The early integrated waveguide front-end system and pre-amplifier of SG-II (AMP: amplifier). 


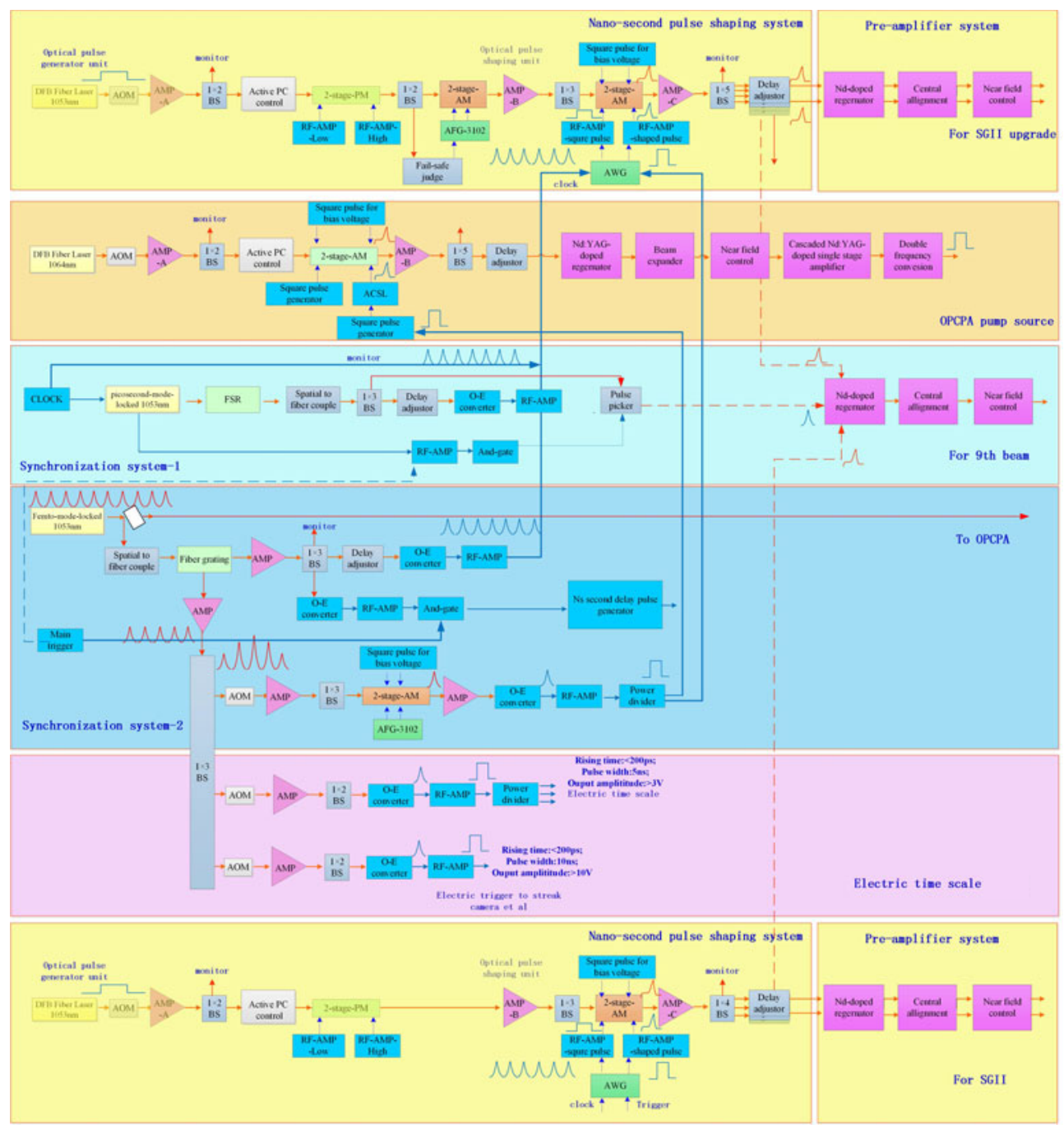

Figure 3. The function of the injection laser system of SG-II.

To meet the demands of the SG-II facility, the injection laser system has numerous functions which can be seen in Figure 3.

The injection laser system of the SG-II provides 9 beams to the SG-II facility. The front end provides 6 beams of nanosecond shaped laser pulses for the SG-II series operated at $1053.02 \mathrm{~nm}$. This is the center of the gain spectrum of the $\mathrm{Nd}^{3+}$-doped amplifier, which is utilized to decrease the FMto-AM effect (amplitude modulation caused by frequency modulation $)^{[22]}$. Two nanosecond beams are used for the SGII and SG-II upgrade. The fifth and sixth nanosecond shaped pulse provides a $100 \mathrm{ps}-25 \mathrm{~ns}$ long pulse for the 9th beam, which allows it to act as a diagnostic laser to separately serve the SG-II and SG-II upgrade. The 7th beam provides a $30 \mathrm{ps} / 80 \mathrm{ps}$ laser pulse for the 9th beam, and also acts as a diagnostic laser. The 8 th beam provides a nanosecond single frequency pump source for the optical parametric chirpedpulse amplification (OPCPA), which is part of the front end of PW system. The 9th beam was also used to provide the mode-locked short pulse seed source for the OPCPA.
The synchronization unit is used to facilitate the synchronization between short and long shaped pulses.

In addition, the injection laser system provides optical and electric timescales for physical experiments.

In order to achieve laser characteristics control, we developed several technologies including frequency domain control, time domain control, spatial shaping control and preamplifier technologies since the beginning of the SG facility.

The specifications of the SG-II injection laser system are shown in Table 1.

\section{The progress of key technologies}

\subsection{Frequency domain control technology}

The frequency domain control unit addresses three critical issues. The first is the acquisition of a single frequency laser output to achieve a smooth waveform in time domain. The second is the broadening of the spectrum to decrease 
the spectral power density in order to suppress the transverse stimulated Brillouin scattering (TSBS) effect. This phenomenon may cause damage to the large aperture optics components. The third is the broadening of the spectrum with high-frequency to meet the demands of focal spot smoothing by spectral dispersion.

In order to acquire smooth waveforms, the single frequency laser was used to minimize interference modulation $^{[18,19,23]}$. The bandwidth of the single frequency is less than $100 \mathrm{kHz}^{[11]}$ and it induces TSBS at the large aperture optics which results in damage ${ }^{[24,25]}$. Therefore, the single frequency, narrow bandwidth laser should be broadened to at least $0.1 \mathrm{~nm}$ to suppress TSBS and to protect the large aperture optics component. This can be accomplished using a phase modulator ${ }^{[1,11,26,27]}$.

In addition, in order to provide a smooth focal spot in a predetermined integration time, such that the instability invoked by laser-plasma interaction (LPI) is suppressed, a high-frequency phase modulation technology was developed $^{[1,26-29]}$. Moreover, two-dimensional SSD ${ }^{[30,31]}$ or multi-frequency modulation was investigated in this $\operatorname{regard}^{[32,33]}$.

Figure 4(a) is the spectrum of the single frequency laser output. Figure 4(b) represents the broadened spectrum with
$3 \mathrm{GHz}$ phase modulation, which was used to decrease the spectrum power density to avoid TSBS. Figure 4(c) is the broadened spectrum with $22 \mathrm{GHz}$ phase modulation, which was used to increase the scan velocity of the focal spot to realize a smooth spot during $\sim 50$ ps time interval. This is determined by the bandwidth of the phase modulator. Waveguide phase modulators are matured products. However, for LPIs, Lin et al. determined that the observed nonlinear effects are mainly caused by self-focusing ${ }^{[34]}$. If the timescale of the self-focusing is approximately $10 \mathrm{ps}$, then the time scan velocity should be lower than this interval to prevent a nonlinear effect and to maintain a smooth spot for the timescale of the fusion ignition. This means that the bandwidth of the phase modulator should be more than $100 \mathrm{GHz}$, which is not yet practically achievable.

Multi-dimensional spectrum dispersion smoothing would further improve the focal spot smoothing ${ }^{[31,35]}$. The highfrequency bulk phase modulator was developed by our team, as a key component of multi-dimensional spectrum dispersion $^{[35]}$.

\subsubsection{The bulk modulator}

One of the most important parameters for evaluating the efficiency of high-frequency bulk phase modulators is the modulation depth (Table 2). The lower the half-wave voltage,

Table 1. The specifications of SG-II.

\begin{tabular}{|c|c|c|}
\hline & Specifications & SG-II series \\
\hline \multirow{7}{*}{ Near-field distribution control ability } & Contrast & $>500: 1$ \\
\hline & Precision & $\pm 3 \%(\mathrm{PV}, 120 \mathrm{ps})$ \\
\hline & Rising edge & $100 \mathrm{ps}$ \\
\hline & Waveform stability & $2 \%(\mathrm{rms})$ \\
\hline & Duration & $0.1-25 \mathrm{~ns}$ \\
\hline & Damage threshold & $\begin{array}{l}7 \mathrm{~J} / \mathrm{cm}^{2} \\
(1064 \mathrm{~nm} @ 12 \mathrm{~ns})\end{array}$ \\
\hline & Resolution & $12 \mu \mathrm{m} \times 12 \mu \mathrm{m}$ \\
\hline Near-neld distribution control ability & Damage threshold & $\begin{array}{l}100 \mathrm{~mJ} / \mathrm{cm}^{2} \\
(1064 \mathrm{~nm} @ 12 \mathrm{~ns})\end{array}$ \\
\hline Optical addressed spatial modulator & Resolution & $20 \mu \mathrm{m} \times 20 \mu \mathrm{m}$ \\
\hline \multirow{3}{*}{ High-gain regenerative amplifier } & Energy & $12 \mathrm{~mJ}$ \\
\hline & Stability & $1 \%(\mathrm{rms})$ \\
\hline & Square-pulse distortion & 1.5 \\
\hline Spectrum control & Bandwidth & $0.1-0.3 \mathrm{~nm}$, with fail-safe feedback control \\
\hline Synchronization & Precision & 20 ps (PV); 3 ps (rms) (2 hours) \\
\hline FM-to-AM control & $\begin{array}{l}\text { Transmission spectrum } \\
\text { tuning ability }\end{array}$ & $\begin{array}{l}1053 \pm 0.5 \mathrm{~nm} \text {; transmission rate } \\
\text { tuning ability } \sim 30 \mathrm{~dB}\end{array}$ \\
\hline Repetition $\mathrm{Nd}^{3+}$ doped amplifier & Energy and wavefront & $1 \mathrm{~J}(1 \mathrm{~Hz}), 0.3 \lambda$ \\
\hline
\end{tabular}

Table 2. The key parameters of the bulk phase modulators of SG-II and OMEGA.

\begin{tabular}{|c|c|c|c|c|}
\hline \multirow{2}{*}{$\begin{array}{l}\text { Parameters } \\
\text { Band frequency }(\mathrm{GHz})\end{array}$} & \multicolumn{2}{|r|}{ SG-II } & \multicolumn{2}{|c|}{ OMEGA $[36,37]$} \\
\hline & 3.25 & 10.302 & 3.3 & 10.412 \\
\hline Aperture (mm) & $5 \times 5$ & $3 \times 2$ & $5 \times 6$ & $3 \times 2$ \\
\hline Modulation spectrum bandwidth & $0.12 \mathrm{~nm} / 1 \mathrm{~kW}$ & $0.67 \mathrm{~nm} / 300 \mathrm{~W}$ (double stage) & $0.15 \mathrm{~nm} / 3.5 \mathrm{~kW}$ & $1.1 \mathrm{~nm} / 300 \mathrm{~W}$ (double stage) \\
\hline Modulation depth (rad/V) & 0.025 & 0.072 & 0.015 & 0.118 \\
\hline
\end{tabular}





Figure 4. (a) The spectrum of a single frequency output; (b) the broadened spectrum with $3 \mathrm{GHz}$ phase modulation; (c) the broadened spectrum with $22 \mathrm{GHz}$ modulation; (d) the broadened spectrum with $3 \mathrm{GHz}+22 \mathrm{GHz}$ modulation.

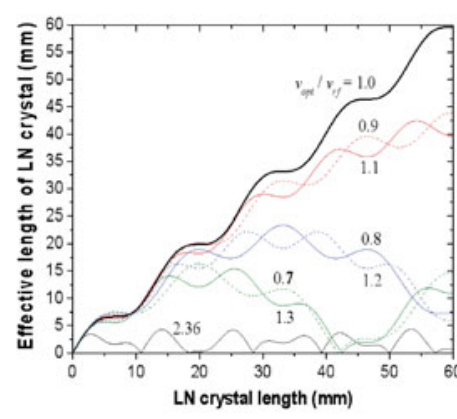

(a)

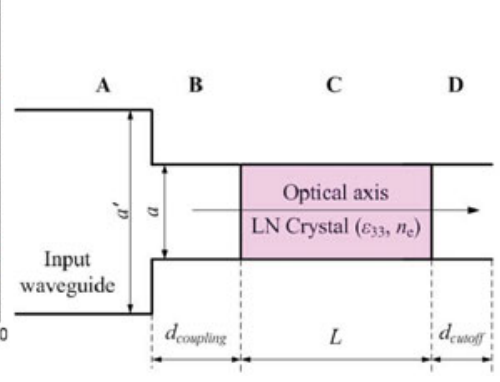

(b)

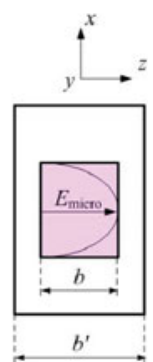

Figure 5. (a) The relationship between the effective length and the actual length of the crystal under different velocity matching conditions (the design frequency is $10.5 \mathrm{GHz}$, the crystal is lithium niobate). (b) The structure of the resonant cavity modulator based on the cut-off waveguide (A: the injection waveguide; B, D: cutoff waveguide; C: electro-optic crystal).

the higher the modulation depth value, and the higher the modulation spectral bandwidth that can be obtained under the same driving power. To improve the efficiency of the bulk modulator, there are three key problems that should be addressed.

(1) Velocity matching. In the high-frequency band, light waves and micro-waves should achieve phase velocity match; otherwise, the modulation efficiency will be adversely affected. Figure 5 shows the simulation results of the relationship between the effective length and the actual length of the crystal under different velocity matching conditions.

(2) Micro-wave coupling efficiency. In the high-frequency band, a special structural design is required to ensure high coupling efficiency.

(3) $Q$ factor. The micro-wave loss in the modulator is proportional to the $Q$ factor when other conditions are constant.
A cut-off waveguide based on the resonator cavity modulator design was adopted and the structure is shown in Figure 5 on the right. The high-efficiency high-frequency bulk phase modulator was finally developed by effectively addressing the aforementioned three technical challenges.

(1) In the case of the uncertainty of the dielectric constant $\left(\varepsilon_{33}\right)$ of the lithium niobate crystal material in the modulator (which was reported to be between 23 and 30), the key parameters of the modulator microwave resonator were designed based on theoretical simulations and experimental data. In the process, the phase velocity of the light waves and micro-waves was determined. The micro-waves of the $3.25 \mathrm{GHz}$ modulator work in the $\mathrm{TE}_{101}$ mode, while the microwaves of the $10.302 \mathrm{GHz}$ modulator work in the $\mathrm{TE}_{104}$ mode. Compared to the bulk modulator based on the domain inversion of materials, the latter achieves quasi-velocity matching and is restricted to the processing technology of the crystal domain structure. In 


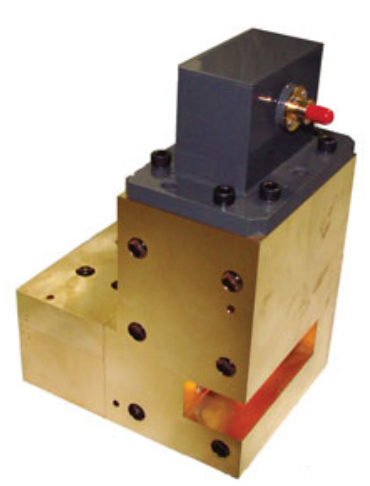

(a)

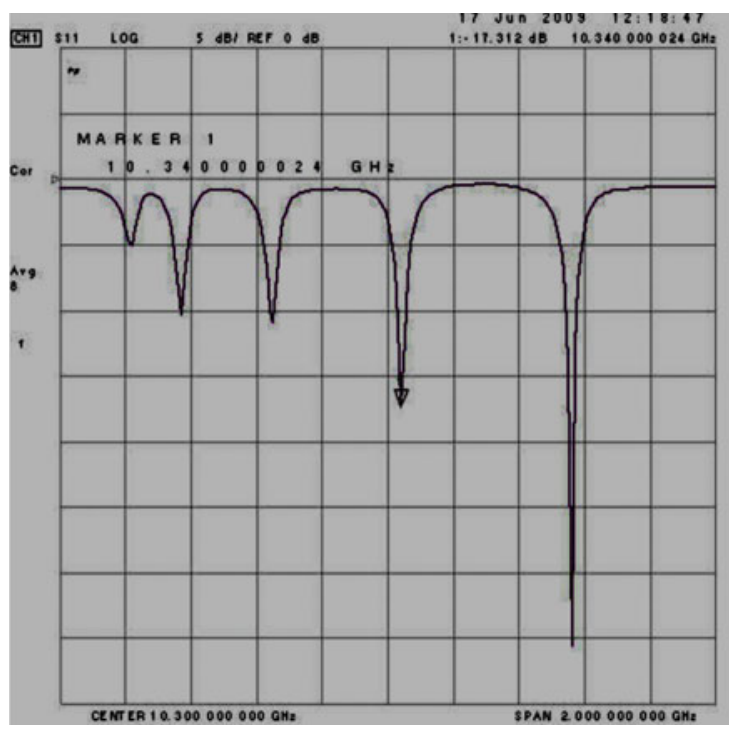

(b)

Figure 6. (a) The bulk modulator prototype. (b) $S_{11}$ curve of $10.302 \mathrm{GHz}$ bulk modulator.

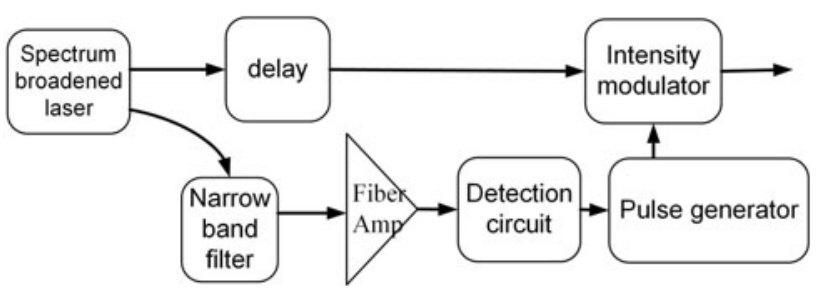

(a)

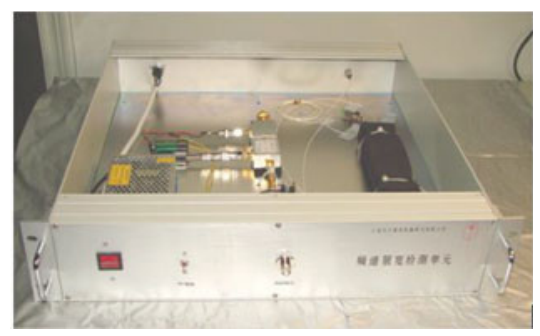

(b)

Figure 7. (a) The illustration and (b) physical map of the fail-safe system.

this case, an aperture size of $\sim 1 \mathrm{~mm}$ was obtained for the modulator.

(2) By using the structure based on cut-off waveguide coupling, the gold-plated electrode of the crystal is optimized and the micro-wave coupling efficiency of the modulator is greatly improved. In particular, $S_{11}$ is less than $-15 \mathrm{~dB}$. The result for this structure is significantly better than the result for micro-strip coupling, for which $S_{11}$ is usually around $-4 \mathrm{~dB}$. Approximately half of the micro-wave power is reflected, and micro-wave utilization is low, which threatens the long-term safe use of the micro-wave drive source.

(3) Due to the successful application of the aforementioned cut-off waveguide-coupled resonant cavity structure, it is ensured that the modulator has a high $Q$ factor. This results in a high modulation depth value (i.e., a lower half-wave voltage) for the modulator in the case of a larger aperture. The $Q$ value is much higher than that of the traveling wave structure of the bulk modulator.
We have developed high-frequency bulk phase modulators with $3.25 \mathrm{GHz}$ and $10.302 \mathrm{GHz}$ modulation frequencies, a maximum modulation bandwidth of approximately $6.7 \AA / 240 \mathrm{~W}(1 \AA=0.1 \mathrm{~nm})$, and a maximum aperture of approximately $5 \mathrm{~mm} \times 5 \mathrm{~mm}$ (Figure 6).

\subsubsection{Fail-safe system for the spectrum broadening}

To avoid damage to the large diameter optical components caused by the TSBS under high-energy density ${ }^{[38]}$, there are two technical difficulties. The first is that discrimination between the spectrally broadened signal and the unmodulated signal is challenging. The second is the timely generation of the control signal for the subsequent device to ensure that the shut-off time of the laser is sufficiently short when spectrum broadening fails.

In order to shut down the laser in case the spectrum broadened system failed, monitor and safety equipment were developed by our team to improve the operational safety of the high power laser facility.

In this equipment, a narrow-band filter was used to monitor the laser spectrum, as shown in Figure 7. Since this filter has different transmission values for different wavelengths, 


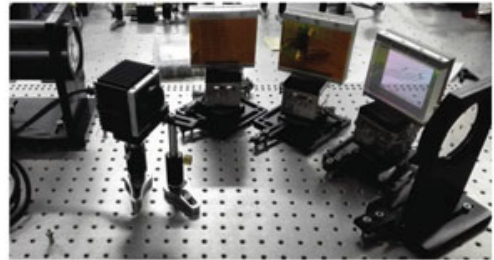

(a)

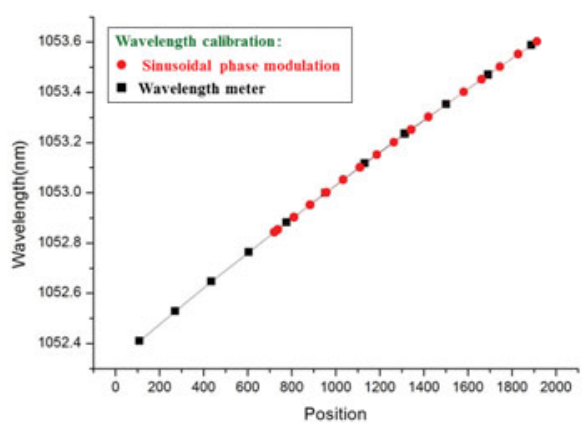

(b)

Figure 8. (a) High-resolution single-shot spectrometer prototype; (b) the calibration results with the wavelength meter.

it causes an FM (frequency modulation) to AM (amplitude modulation) effect ${ }^{[39]}$. Therefore, the AM can be used to monitor whether a single frequency laser was broadened or not. As soon as the AM is detected, the fail-safe system generates an output signal to control the on or off state of the optical gate of the laser system to avoid damage to the large aperture optical components as shown in Figure 7. The response time is $\sim 200 \mathrm{~ns}$, and the signal travels along a transmission delay fiber in the main laser path as shown in Figure 7.

\subsubsection{High-resolution single-shot spectrometer}

The laser spectrum needs to be accurately tested for accurate frequency control. However, it is difficult to obtain highresolution spectral information for a single-shot laser pulse using currently available commercial spectrometers.

A set of gratings was used to build such a spectrometer by our team, which can be sampled using a fiber as shown in Figure 8.

The key parameters of the single-shot spectrometer include:

- center wavelength: $1053 \mathrm{~nm}$;

- range: $1.2 \mathrm{~nm}$;

- resolution: 3 pm.

The spectral width of the front-end system was measured by the spectrometer as shown in Figure 9 .

The successful development of a high-resolution singleshot spectrometer provides an important means of testing the spectral control techniques of high power laser devices.

\subsection{Time domain control technology}

Time domain control technology includes synchronization and waveform (that is the time-power curve) control.

For synchronization control, there are two technology routes. The first is the optically driven synchronization technology. The second is the homologous clock-lock, phaselocked frequency synchronization technology.

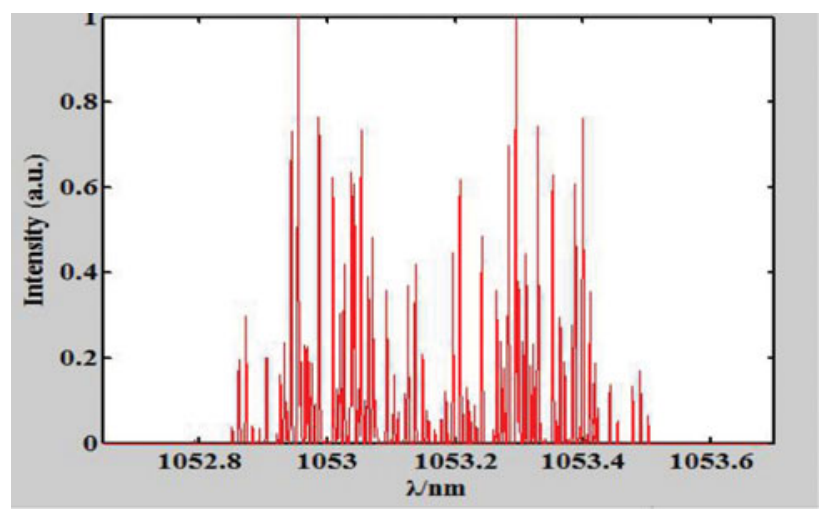

Figure 9. The laser spectrum (bandwidth is $0.52 \mathrm{~nm}$ ) which was measured using a home-made single-shot spectrometer.

For the waveform control, a single-longitudinal-mode laser with amplitude waveguide modulator was utilized to achieve smooth waveform shaping. The FM-to-AM effect, which is caused by polarization-mode dispersion and group velocity dispersion, needs to be effectively controlled during the process.

\subsubsection{The synchronization control technology}

4.2.1.1. Optical driven synchronization technology. The high-precision and high-stability synchronization performance of different laser beams is a key requirement for active diagnosis, fast ignition research and high-efficiency implosion studies. Therefore, accurate synchronization of different laser beams is one of the most important technical indicators of the front-end subsystem of the SG-II. Optically triggered synchronization technology was proposed to achieve precise synchronization between hundred picosecond pulses and nanosecond laser pulses ${ }^{[11,21]}$. The main difficulty involves obtaining a sufficiently large amplitude, width and fast-rising edge for the high stability trigger signal so that the shaping pulse unit works in a highly stable time trigger state.

For synchronization in high power laser systems, not only is the synchronization between the main nanosecond laser pulse and the diagnostic picosecond pulse involved, but also 


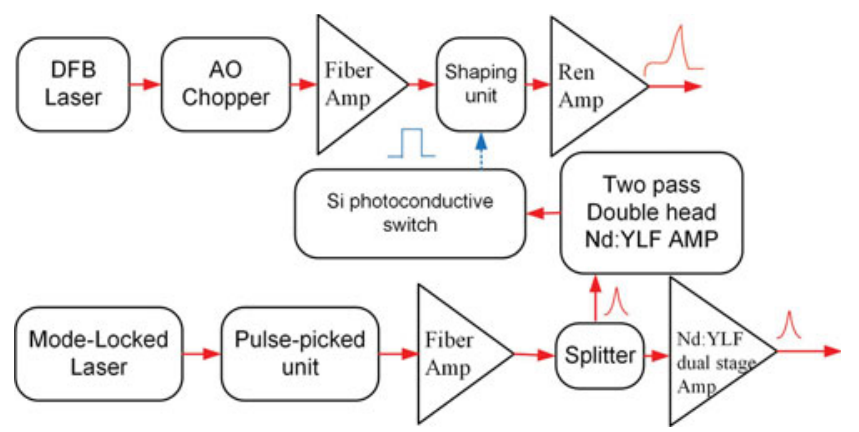

Figure 10. The early synchronization scheme of SG-II between the nanosecond shaped laser and the short pulse picosecond laser.

the synchronization between the main laser pulse and the diagnostic instrument.

In the SG-II series, dual oscillators were separately used as laser sources for the main laser and the diagnostic laser. Optical trigger technology was used to achieve synchronization. In the early SG-II, the optical trigger module included a four-stage amplifier and a $\mathrm{Si}$ photoconductive switch, as shown in Figures 2 and $10^{[11,21]}$. The short pulse laser was initially split into two beams. One beam was used as the short pulse source, and the other was amplified by a twopass double head Nd:YLF amplifier. This device can output a micro-joule laser signal to trigger the Si photoconductive switch to produce an electrical pulse greater than $7 \mathrm{~V}$ to drive the nanosecond pulse shaping system. Using this technology, pulse synchronization of $15.7 \mathrm{ps}(\mathrm{PV})$ for 10 shots can be achieved.

However, during operation, synchronization is not stable over the long term and this process can degrade to as high as $400 \mathrm{ps}(\mathrm{PV})$, due to the instability of the twopass double head Nd:YLF amplifier. This directly depends on the stability of the picosecond laser and the coupling injection stability. In addition, since the output electrical pulse amplitude is linearly dependent on the output of the Nd:YLF amplifier, this signal is also not stable. When the trigger time for the nanosecond shaping system is changed, this influences the synchronization between the short pulse laser and the shaped nanosecond laser. So the stability of the short pulse laser and Nd:YLF amplifier has a great influence on the synchronization stability of the main and diagnostic pulses.

In order to solve this problem, the fiber stacker, high-speed electronics, and analog to digital conversion technology were adopted as shown in Figure 11. This greatly decreases the dependence of the trigger module on the input laser stability. Thus, the long-term synchronization stability between the main pulse and the diagnostic pulse was improved ${ }^{[40,41]}$.

To test the level of synchronization, a $3 \mathrm{~dB}$ coupler was used to couple the shaped laser pulse and the short laser pulse to the same fiber. This signal was converted into an electrical signal using PIN(GD4961T) and tested using an oscillator

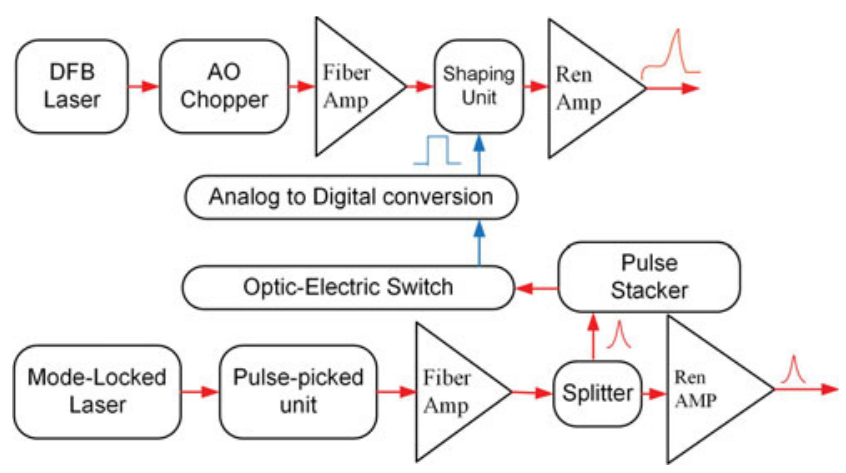

Figure 11. Improved optically driven synchronization schematic.

(Tek,TDS6124C). The acquired data is shown in Figure 12. Synchronization was less than $4.1 \mathrm{ps}$ (rms) over a period of 2 hours and less than $3.2 \mathrm{ps}$ (rms) for 4 minutes. The synchronization module has been used in the SG-II series facility for more than 5 years.

4.2.1.2. Homologous clock-lock, phase-locked frequency synchronization technology. Since the optical-triggered synchronization technique is limited by the stability of the mode-locked laser sequence, changes in the cavity length of the mode-locked laser caused by temperature shifts lead to changes in the repetition frequency (time interval between pulses). Thus, the long-term stability of the system's long and short pulse synchronization accuracy is affected. Thus, a homogeneous clock lock, which clamps the laser frequency of the high-precision synchronization program was proposed as shown in Figure 13. The main clock is used to lock the mode-locked laser, the high-frequency phase modulation signal and arbitrary electric waveform generator (AWG) within the clock signal to achieve long pulse synchronization accuracy of $<3$ ps (rms), peak and peak $<20$ ps ( 2 hours test results), which has been applied in the SG-II ${ }^{[41]}$.

Physical experiments require high-precision synchronous trigger signals, in addition to optical and electric scale signals, for multi-channel physical test diagnosis. The electric scale signals produced by the pulse picker and the opticalelectrical conversion of the femtosecond laser pulse signal are shown in Figure 2. The optical scale signals originated from the single-longitudinal laser with the intensity modulator and the electric pulses originated from the AWG of the main laser system. The time jitter between the signals and the main laser is $\leqslant 3 \mathrm{ps}(\mathrm{rms}), 20 \mathrm{ps}(\mathrm{PV})$, and a certain amount of delayed tuning is satisfied in the process.

4.2.2. The optical pulse shaping technology of injection shaped laser sources

In order to acquire smooth laser pulse envelopes, the front end of the SG-II adopted an integrated waveguide modulator shaping system in combination with an electrical waveform generator $^{[17,20,41-43]}$, as shown in Figure 14. The pulse shaping system requires that the pulse shaping is controlled 


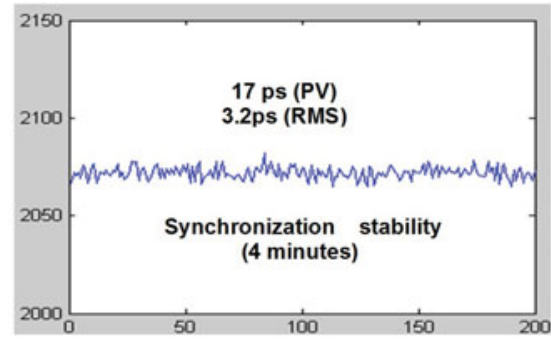

(a)

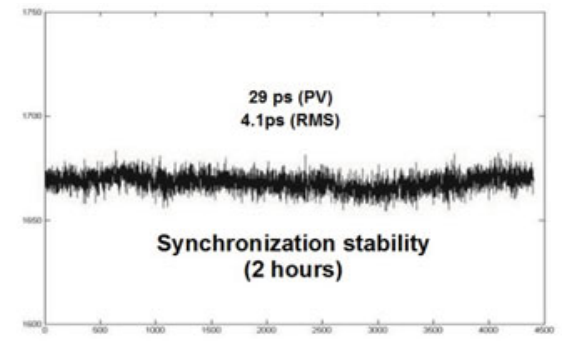

(b)

Figure 12. Synchronization stability testing results for (a) 4 minutes and (b) 2 hours between the nanosecond laser and the picosecond pulse laser.

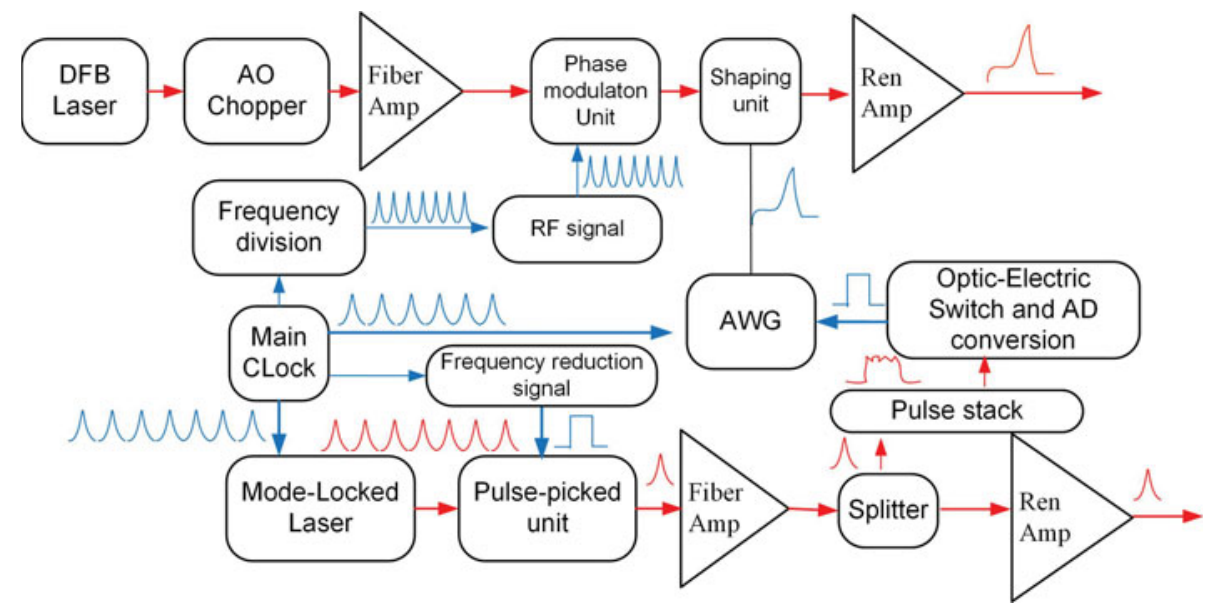

Figure 13. Homologous clock-lock, phase-locked frequency synchronization scheme.

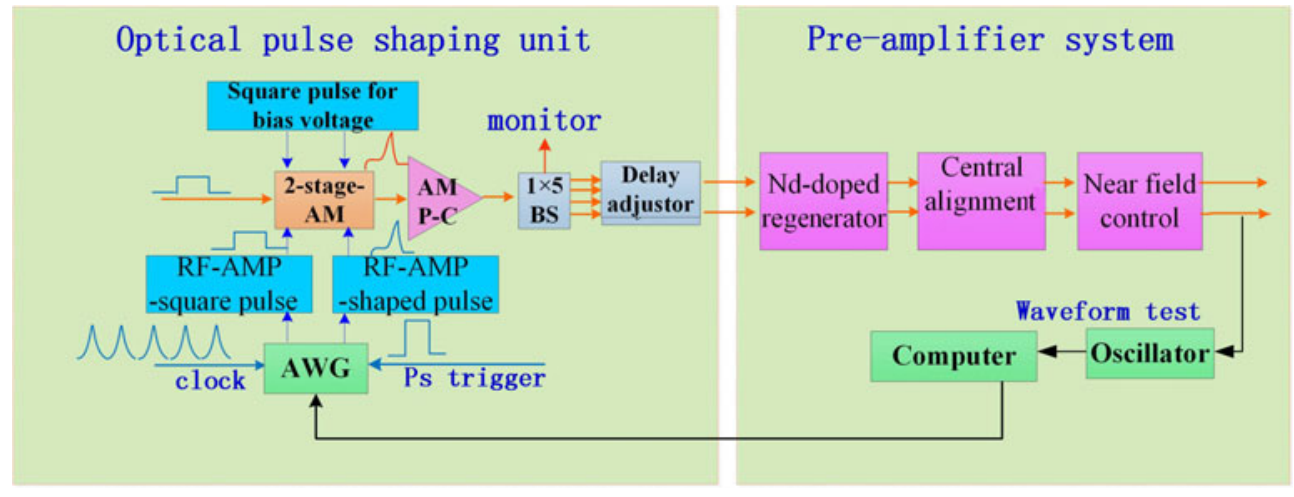

Figure 14. The temporal pulse shaping schematic.

actively and in a flexible manner with minimal delay. For the SG-II series, the pulse shaping system uses a drag four form, that is, one beam of the injection laser system including the front-end and pre-amplifier system is divided into four before injected into the main amplification system.

The integrated waveguide modulator can be controlled using an electric waveform generator. There are three kinds of electric waveform shaping technologies. The first is electric pulse stacker ${ }^{[43,44]}$, which is based on a micro-wave GaAs-FET, but ripple is a problem and the precise pulse shape near the peak of the pulse cannot be maintained ${ }^{[44]}$.
The second is the aperture-coupled strip line (ACSL), which consists of two strip-lines coupled through an aperture in their common ground plane $e^{[11,20,41,42]}$. The ACSL pulse shaping technology can accurately acquire stable laser pulse envelopes without ripple waves for $120 \mathrm{ps}-3 \mathrm{~ns}$. The longest pulse can be as much as $8 \mathrm{~ns}^{[11]}$. However, for the longer pulses, it was difficult to reach the same level of precision. In addition, because the strip line must be processed by photo etching, several days are needed to change the laser pulse waveform. The third is arbitrary electrical waveform generator based on field programmable gate array (FPGA) 


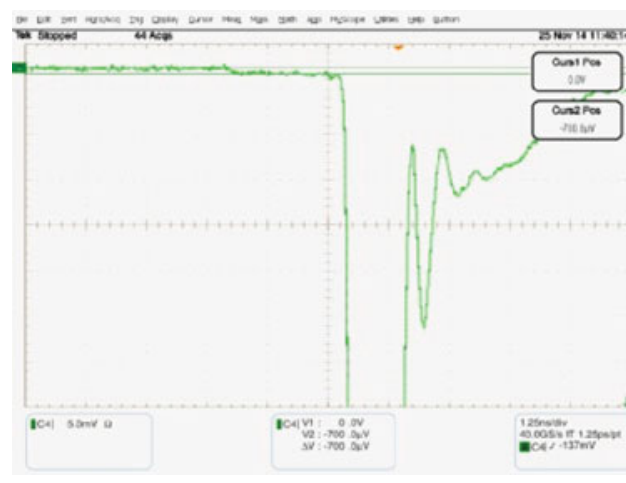

(a)

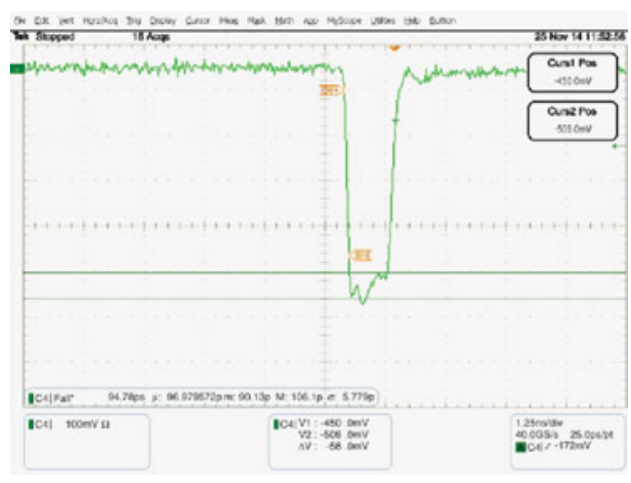

(b)

Figure 15. The high contrast temporal waveform (600:1). (a) Low amplitude pedestal; (b) high amplitude step.

technology. This approach is sufficiently flexible to be able to adjust the waveform online in real time. However, because the output voltage of the AWG is too small to reach the half-wave voltage of the waveguide modulator, a high-speed electric amplifier must be used and the linear characteristics of this device must be considered. The $4 \mathrm{G} \mathrm{RF}$-amplifier is used to achieve more agile waveform shaping and the $10 \mathrm{G}$ RF-amplifier is used as the gate pulse amplifier to increase the rise edge.

Using the 2-stage intensity modulator, different kinds of output temporal waveforms can be acquired. The maximum contrast ratio can be greater than 600:1 as shown in Figure 15 and the rising edge of the front edge is $\sim 100 \mathrm{ps}$. The test device includes an oscillator (TDS6124C) and a highspeed photodetector (GD4961T).

One of the problems with the approach is the drift of the bias voltage of the intensity modulator, which influences the signal-to-noise ratio of the laser. Shortening the pulse width of the bias voltage load on the amplitude modulator may alleviate the problem. In addition, a separate unit to monitor the signal-to-noise ratio of the intensity modulator in case of drift of the bias voltage should be constructed.

The waveform can be controlled using a closed loop with feedback control as shown in Figure 14. The output of the regenerative amplifier is sampled by the photoelectric tube and the high-speed oscilloscope to measure the pulse waveform. Real-time remote acquisition of the pulse waveform data measured by the oscilloscope is processed and the computer automatically feedbacks the AWG shaping output.

In the defined operating state (AWG sampling frequency, the bias voltage of the electrical amplifier), the state of the pulse shaping system is first calibrated, including the output characteristic curve of the amplitude modulator and the amplification characteristics of the electric amplifier. According to the required regenerative output waveform, the regenerative injection pulse waveform is obtained by solving the inverse problem of regenerative amplification. According to the output characteristic curve of the amplitude modulator and the amplification characteristic curve of the electric amplifier, the AWG shaping electric pulse is obtained as the initial condition of pulse shaping.

The closed-loop control is adopted in the shaping process to reduce the influence of noise, calibration error, and nonlinearity of the electric amplifier on the shaping precision. Each iteration process varies the pulse voltages according to the deviation between the target power and the measured power.

$$
\begin{aligned}
V_{j}^{\prime} & =V_{j} \frac{G_{j}}{M_{j}} \\
\Delta V_{j} & =V_{j}^{\prime}-V_{j}=V_{j}\left(\frac{G_{j}}{M_{j}}-1\right) g,
\end{aligned}
$$

where $V_{j}$ is the sub-pulse voltage, the target power is $G_{j}$ and the measured power is $M_{j}$. Due to the nonlinearity of the actual intensity modulator photoelectric response, the voltage correction factor $g$ is introduced. The sub-pulse voltage change is modified as Equation (2).

The correction factor $g$ in this iterative process is dynamically adjusted with the change of the target powers $G_{j}$ and $G_{j} / M_{j}$. In the pulse shaping process, the iterative process is repeated until the convergence criterion is implemented. In the iterative process, the integral error $E$ is expressed as

$$
E=\sum_{i=0}^{n} M_{i}-G_{i}
$$

$M$ is the measured waveform, $G$ is the target waveform, and the standard deviation $\sigma$ of the integral error of the convergence criterion for 6 times is usually less than the threshold value, where $\sigma$ can be expressed as

$$
\sigma=\sqrt{\frac{1}{n} \sum_{i=1}^{n}\left(E_{i}-\bar{E}\right)^{2}}, \quad n=6 .
$$

The following figure shows the results of Haan pulse shaping. For the case of the pre-injection waveform and 


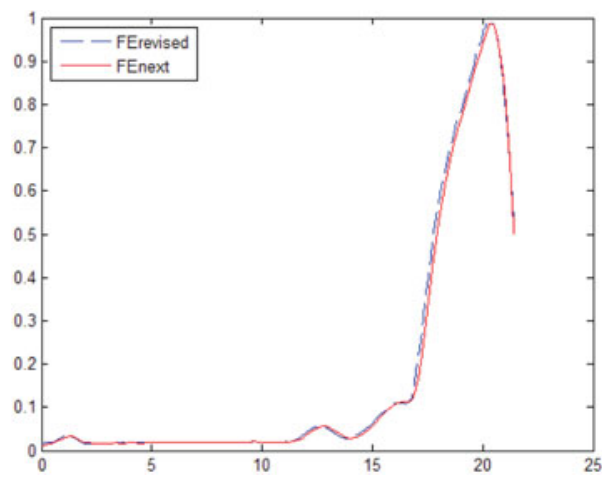

(a)

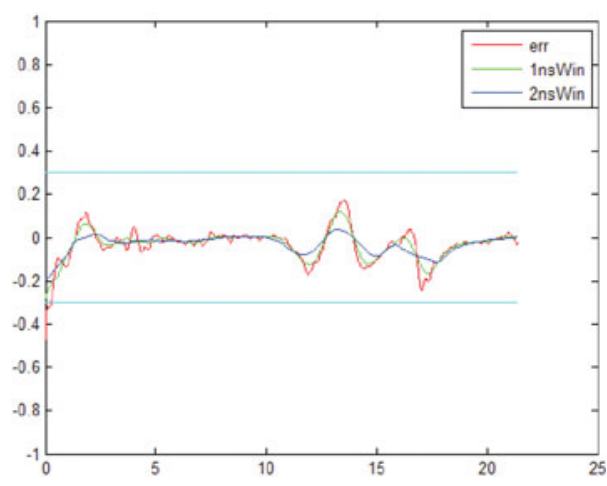

(b)

Figure 16. (a) Pre-placed injection waveform and (b) AWG closed-loop deviation.

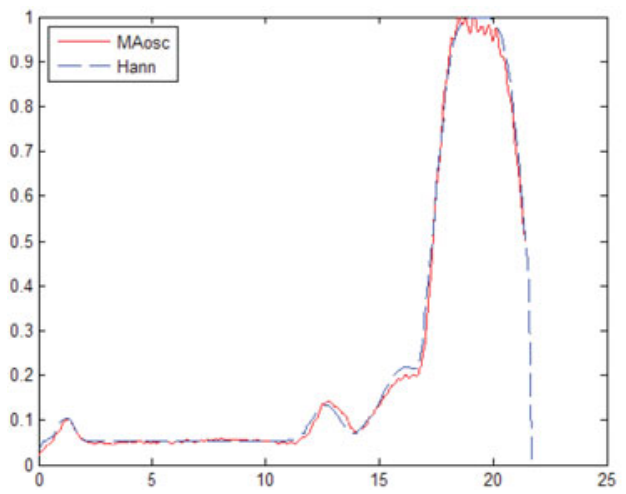

(a)

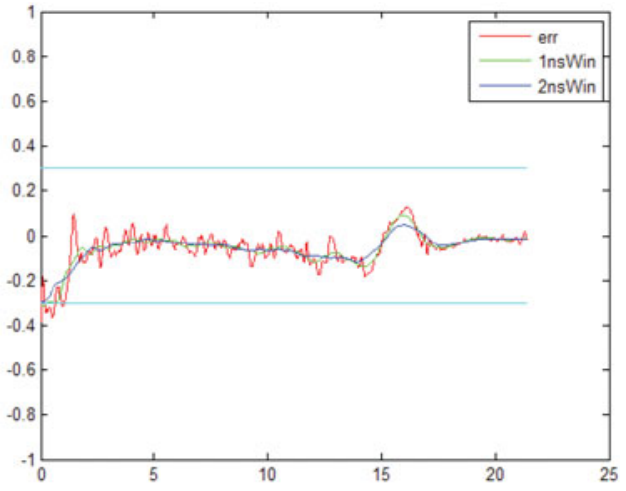

(b)

Figure 17. (a) The main amplifier output waveform; (b) the actual output and the expected output deviation.

AWG closed-loop deviation with the use of a 2 ns window error analysis, the closed-loop pulse waveform deviation is less than $10 \%$ and $3 \%$ separately within the entire window, and within the main pulse as shown in Figure 16.

Figure 17 represents the output waveform at the end of the main amplifier (Figure 17(a)) and the deviation between the actual output and the expected output (Figure 17(b)). Using a 2 ns window error analysis, the time waveform deviation in the entire window can be controlled within $10 \%$, with a main pulse deviation of less than $3 \%$.

\subsubsection{FM-to-AM effect suppression technology}

Due to the need to suppress the TSBS to protect the large aperture optics and for uniformity of the focal spot, spectrum broadened technology was used in the high power laser systems, such as the NIF, OMEGA, LMJ and SG series $^{[1-5,9-11,24-29]}$. However, after spectral broadening, the discrepancy of the transmission rate of the spectral phase or amplitude leads to modulation of the time waveform, which is an FM-to-AM effect ${ }^{[22,45-52]}$.

In high power laser systems, the main causes of FM-toAM include group dispersion, polarization-mode dispersion $^{[51]}$, gain narrowing, interference effect caused by weak reflection of the optical components, and harmonic conversion processes ${ }^{[52]}$.

The FM-to-AM effect causes two primary problems for high power laser systems. Firstly, it influences the precise control of the laser waveform, which is the key to the power balance of the laser facility. Secondly, it decreases the safety operation fluency of target optics because of the ability to produce higher power in a short distance, which can destroy the optical components.

In the case of the phase transmission rate discrepancy, a reduction of the transmission dispersion or pre-compensation of the remainder dispersion can effectively address the problem.

There are several different spectrum pre-compensation schemes for the $\mathrm{NIF}^{[1,10,22,48]}$ and $\mathrm{LMJ}^{[50,52]}$.

The NIF adopted a chirp fiber grating to pre-compensate the spectrum dispersion ${ }^{[9]}$. The air F-P (Fabry-Perot interferometer) was used to pre-compensate the gain narrowing effect $^{[9]}$. It is difficult to adjust the F-P adopted by the NIF and it is not stable. According to reports in 2012, 18 hours was required to adjust the pre-compensation when the laser wavelength was changed ${ }^{[48]}$. Polarization-mode dispersion of maintaining-fiber was then used to achieve 


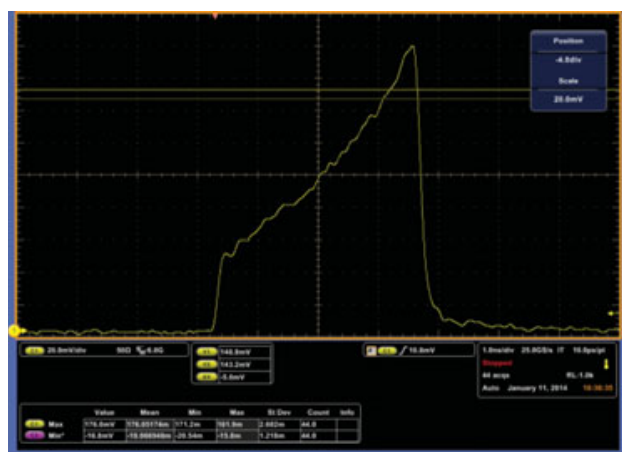

(a)

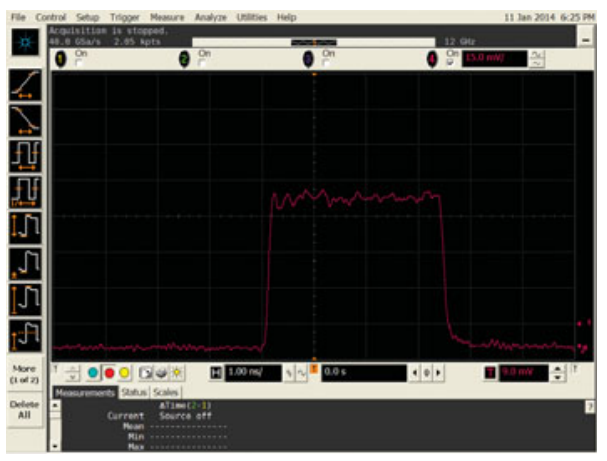

(b)

Figure 18. (a) The output laser waveform of Nd-doped regenerative amplifier and (b) the output laser waveform of one beam of SG-II at $5000 \mathrm{~J}, 1 \omega(0.3 \mathrm{~nm})$.

AM compensation in the 48 beams of the front-end system, which greatly reduced the AM suppression control time ${ }^{[48]}$. In $2016^{[1]}$, the NIF reported that the front end of the system in addition to the gain fiber realized single polarization fiber transmission. Single polarization fiber is characterized by a fast axis cut-off, that is, it acts as an optical fiber polarizer. As the transmission distance increases, the laser optical signal transmitted along the fast axis will dissipate, thus avoiding AM due to polarization-mode dispersion. The NIF reported in October $2016^{[53]}$ on a front-end system gain fiber which also achieved single polarization. Since then, the front-end system of the NIF has fully realized single polarization transmission to fundamentally suppress the FM-to-AM effect due to polarization-mode dispersion. In addition, a birefringent crystal was added to the regenerative amplifier to pre-compensate the spectral loss caused by the gain narrowing of the amplifiers ${ }^{[1]}$. The goal of the NIF is to eliminate the need for active FM-to-AM suppression measures.

The PM fiber was used by the LMJ to maintain a highly stable single polarization output; however, the FMto-AM effect is a serious concern because of polarizationmode coupling ${ }^{[50,51]}$. The polarization dispersion was pre-compensated by controlling the temperature of PM fiber, which has been demonstrated to decrease the FMto-AM effect. The key is that the precision control of the temperature of the PM fiber should reach $0.01^{\circ} \mathrm{C}$. However, for a polarization-maintaining fiber, the coupling between the fiber fast axis and the slow axis is subject to external temperature and stress. Therefore, the AM effect due to polarization-mode dispersion will have random fluctuations. Through theoretical calculations and experimental testing, the FM-to-AM random fluctuations due to polarizationmode coupling of the PM fiber can approximate $20 \%$ [54].

For mercury, a spatial modulator was used to shape the spectral agility, but the pixel structure limited the shaping ability for multi-frequency modulation. In addition, the system is complex for dispersion beams ${ }^{[47]}$.
For the SG-II series high power laser systems, the transmission fiber was a single-mode fiber for $1053 \mathrm{~nm}$, to avoid random FM-to-AM fluctuations caused by the coupling between the fiber fast axis and the slow axis of the PM fiber. The polarization stability of the laser output was controlled by an active polarization controller. In order to easily control the spectrum and fail-safe feedback system, the phase modulator was set between the AO chopper and the pulse shaping unit. Therefore, the spectrum broadened laser was transmitted along more than $50 \mathrm{~m}$ of single-mode fiber before entering into the $\mathrm{Nd}^{3+}$-glass regenerative amplifier. For the polarization dependent discrepancy, the worst AM can be greater than $20 \%$, but this can be reduced to less than $5 \%$ using polarization control.

The FM-to-AM effect was tested using a high current photoelectric tube and an oscillator (4G, DPO70404) at the output of a regenerative amplifier, as shown in Figure 18(a).

At first, there was concern that the gain narrowing and the polarization optics of the pre-amplifier and main amplifier would cause or deteriorate the FM-to-AM effect. The output waveforms of the pre-amplifier and the main amplifier were both tested as shown in Figure 18. The output of the entire laser system operating at $1053 \mathrm{~nm}$ is $5000 \mathrm{~J}$. The spectrum width is $0.3 \mathrm{~nm}$ which was modulated by a $3 \mathrm{G}$ phase modulator. It can be seen that the FM-to-AM effect is less than $5 \%$ (tested by TDS $6124 \mathrm{C}, 12 \mathrm{G}$ ). In addition, the modulation of the first 2 ns was caused by the electrical shaping system, which was verified. Therefore, it was confirmed that the FMto-AM effect caused by gain narrowing and the polarization of the pre-amplifier and main amplifier is small. It was also confirmed that the main FM-to-AM effect was caused by the front end, in particular, the fiber. In order to further decrease this effect, the phase modulator can be positioned after each pulse shaping system to decrease the influence of fiber dispersion. However, this method would increase the cost and the fail-safe complexity. In addition, because the fiber used could not achieve polarization maintaining, the output polarization of the front end would not maintain high 


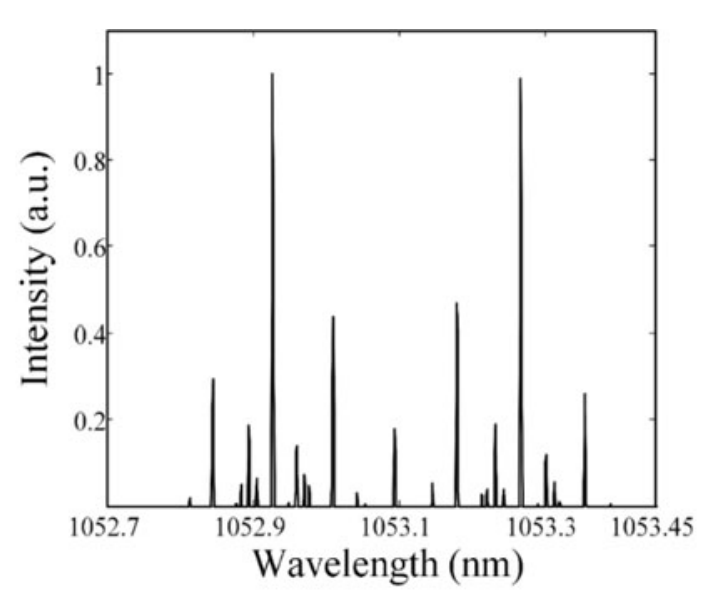

Figure 19. The output spectrum with the $3 \mathrm{GHz}$ and $22 \mathrm{GHz}$ phase modulation.

stability and the FM-to-AM would change with vibration or temperature.

In the 1990s, the 46th Research Institute of China Electronics Technology Group Corporation began to produce single-polarized fiber. In 2016, a home-made singlepolarized fiber was adopted to produce intensity modulators, AO choppers, couplers, wavelength division multiplexers, isolators, filters and other single polarization fiber devices. For the first time in China, a front-end system was built based on single polarization transmission fibers ${ }^{[54]}$. The spectrum output from this system is spectrally broadened by both the phase modulation at $3 \mathrm{GHz}$ and the phase modulation at $22 \mathrm{GHz}$. The widened spectrum is shown in Figure 19.

For comparison, we also built a front-end system based on a polarization-maintaining fiber ${ }^{[54]}$. The AM of this fiber front-end system approaches $20 \%$. In addition, the single polarization front-end AM is $2.3 \%$, as shown in Figure 20 (oscilloscope: Agilent DSO93004L (30 GHz); the high-speed pin tube: Newport, 1014, $45 \mathrm{GHz}$ ). The selfdeveloped AM test device was used to test AM changes in real time, as shown in Figure $21^{[55]}$.
Using automatic FM-to-AM detection technology ${ }^{[55]}$, single polarization transmission and seed source output AM changes are monitored in real time, as shown in Figure 22. The FM-to-AM was $0.95 \%$ (PV) and $0.15 \%$ (rms) within 5 minutes. The FM-to-AM modulation was $2.3 \%(\mathrm{PV})$ and $0.38 \%$ (rms) within 3 hours.

At this point, the front-end system based on a single polarization transmission fiber was used to verify the inhibition of the polarization dispersion and can achieve long-term stability. At the same time, the home-made single polarization fiber device processing technique was also verified.

As such the front end of SG-II with a single polarization transmission fiber can be implemented.

\subsection{Near-field spatial shaping technology}

The spatial shaping system has three functions: the first is to produce a soft aperture to avoid Fresnel diffraction and to act as the initial object surface of the entire laser system; the second is to pre-compensate the spatial dependent gain of the amplifiers; and the third is to pre-block the hot spot of the laser or the damage sites of the large aperture optics. The advancement in near-field spatial shaping technology was used to improve the operating flux level of the high power laser system and to decrease both the construction and the operational cost.

There are several technical difficulties associated with near-field spatial intensity control devices which include: high-resolution spatial intensity control resolution, low wavefront distortion, high transmittance and high damage threshold.

NIF and OMEGA both use serrated masks to act as softedge apertures and binary masks in order to pre-compensate for the spatial dependent gain of the amplifiers ${ }^{[56-59]}$. The serrated and binary masks used was the chromate coat, which has a low damage threshold $\left(\sim 100 \mathrm{~mJ} / \mathrm{cm}^{2}\right)^{[56]}$. Depending on the binary pixelated arrays of metal pixels ${ }^{[56,57]}$ and the high-frequency filtering of the spatial filter, accurate spatial
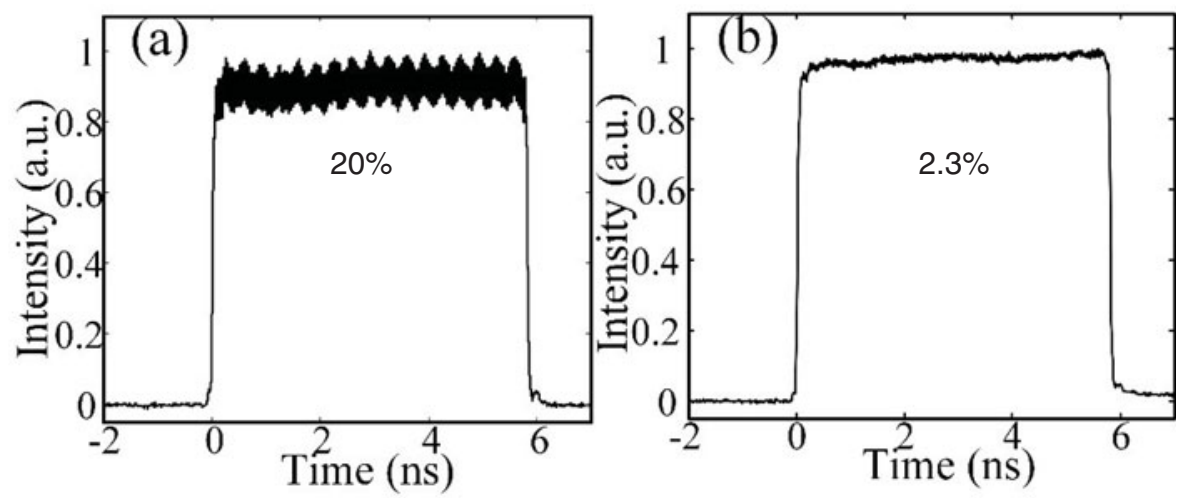

Figure 20. (a) The output waveform of the polarization-maintaining front-end system; (b) the output waveform of the single polarization front-end system. 


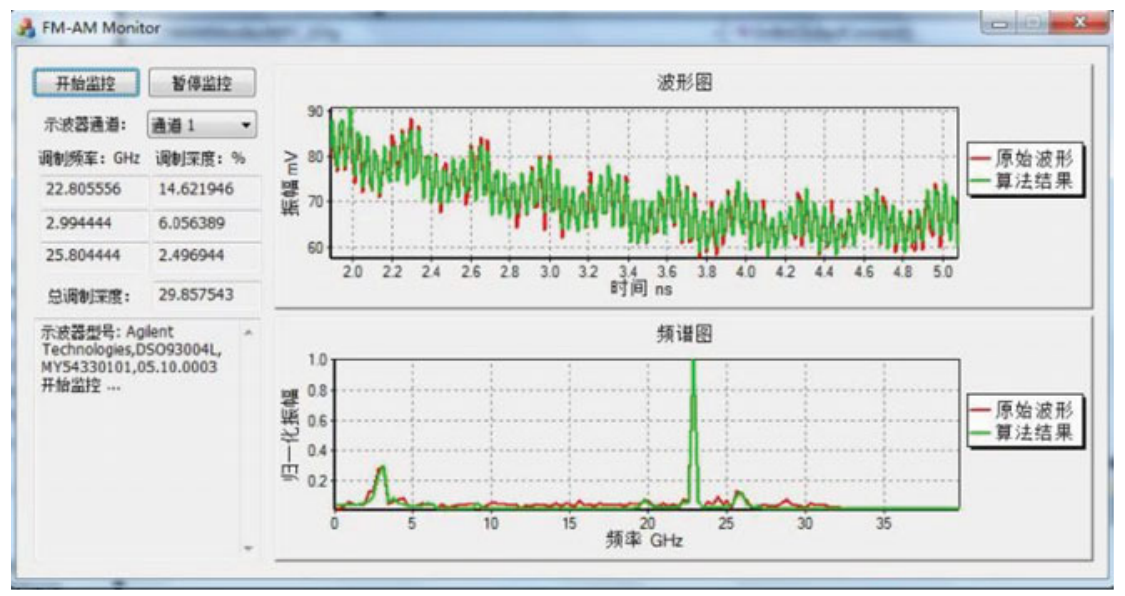

Figure 21. Phase modulation-to-amplitude modulation real-time monitoring software interface.

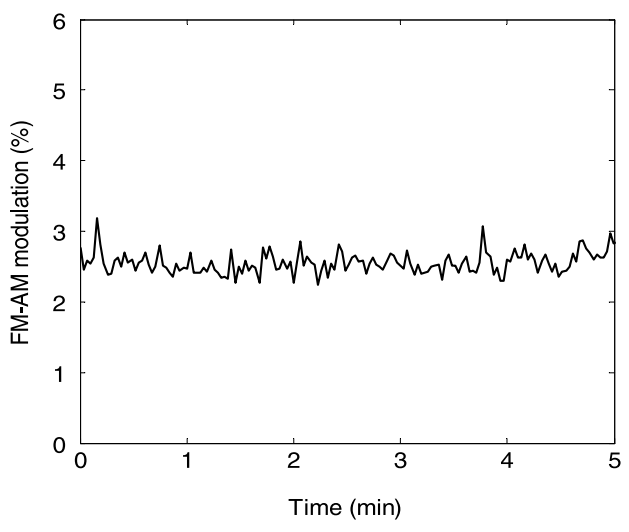

(a)

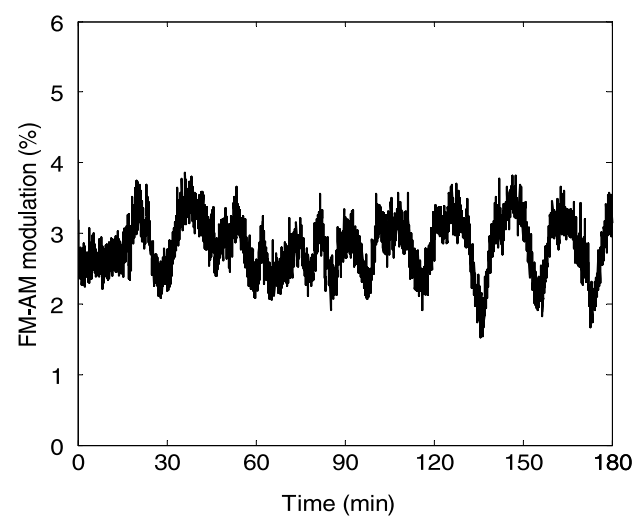

(b)

Figure 22. FM-to-AM changes of single polarization front-end system (a) for 5 minutes and (b) for 3 hours.

intensity distribution can be maintained. In addition, an optically addressed spatial modulator is used to pre-block the defect or damage site of the large aperture optics ${ }^{[60-62]}$.

Given that the damage threshold of the binary mask with the chromate coat is low, we developed high damage threshold binary masks using dielectric films ${ }^{[63]}$. An optical addressed spatial modulator was also developed to control the spatial distribution in real time ${ }^{[64-67]}$.

\subsubsection{High damage threshold binary masks}

The pixel size of the chromate coat binary mask can be as large as $0.3 \mu \mathrm{m} \times 0.3 \mu \mathrm{m}$. If needed, the pixel size can be reduced to $0.1 \mu \mathrm{m} \times 0.1 \mu \mathrm{m}$, which can be readily achieved using lithography techniques. However, the damage threshold is approximately $100 \mathrm{~mJ} / \mathrm{cm}^{2}$, which is too low in most cases for a high power laser system.

The top-hat and super-Gaussian serrated apertures ${ }^{[68-70]}$ made of highly reflecting films have been proposed and fabricated ${ }^{[68-70]}$. In fact, the serrated aperture, as shown in Figure 23(a), has been used in SG-II for more than 20 years ${ }^{[68-70]}$. However, this aperture cannot arbitrarily control the intensity distribution of the output of the system.

As shown in Figure 23(b), we have realized a binary pixel structure with a 1 or 0 transmittance distribution on dielectric film optics glass substrate, for the first time in China ${ }^{[63]}$. The pixel size is $12 \mu \mathrm{m} \times 12 \mu \mathrm{m}$, the damage threshold is higher than $7 \mathrm{~J} / \mathrm{cm}^{2}$, the transmission rate exceeds $99 \%$, the wavefront deviation is less than $0.1 \lambda$, and the aperture can be $50 \mathrm{~mm} \times 50 \mathrm{~mm}$, as shown in Figure 24. Although the unit pixel size is larger than the chromate coat binary mask, it is applicable to near-field spatial intensity control for high power laser systems. In 2016, Rambo also published results for high damage threshold laser-machined apodizers ${ }^{[71]}$.

The primary function of the soft-edge mask which includes more accurate and arbitrary intensity distribution control can be accomplished using a binary mask with the unit pixel arrangement design shown in Figure 25. The binary mask has been used in the SG-II series to act as a softedge aperture and to pre-compensate the spatial dependent gain of the amplifiers. 
(a)

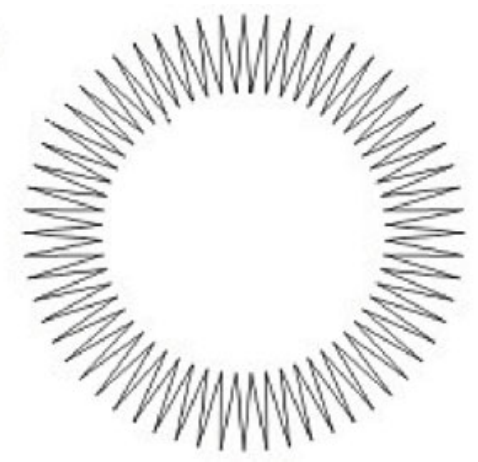

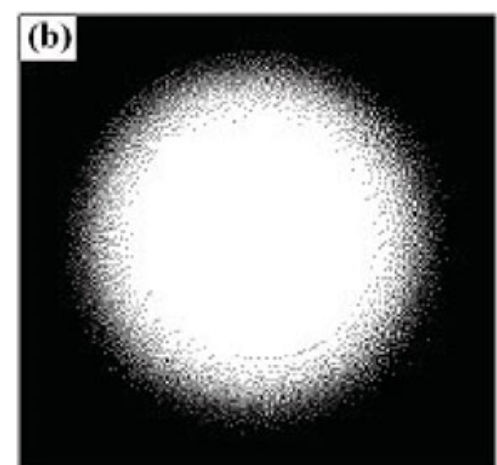

Figure 23. (a) Serrated aperture and (b) binary mask aperture.

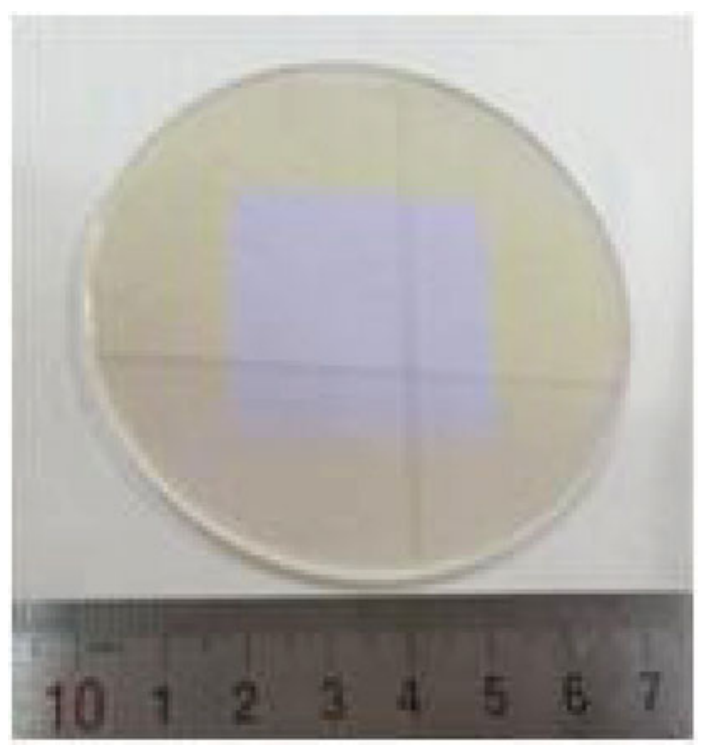

Figure 24. High damage threshold static near-field control element.

In Figure 25(a), the binary mask was used to shape a round Gauss beam to a $10 \mathrm{~mm} \times 10 \mathrm{~mm}$ square, and the beam was subsequently shaped by the binary mask to flat top. It can further be shaped to a parabola distribution, as needed. The device transmission curves and the tested transmission curve are given. It can be seen that the binary mask is able to control the near-field distribution with high accuracy. In Figure 25(b), the laser was shaped to an elliptical near-field distribution using a binary mask in the PW laser system of the SG-II, to meet the aperture of the optical grating to achieve maximum utilization of the laser aperture.

In Figure 26, the binary mask was used in a four-pass $\mathrm{Nd}^{3+}$-doped amplifier setup. The round input laser near-field distribution was shaped by a binary mask to $18 \mathrm{~mm} \times 18 \mathrm{~mm}$, and the gain non-uniformity was pre-compensated to a flattop distribution using an Gauss binary mask.

Since the binary mask has a high damage threshold, it can be used to pre-compensate the damage site at the position of a large optical component with a high-energy flux.
As shown in Figure 27, the high damage threshold binary mask was used in the SG-II upgrade as a second near-field shaping component, to improve the sharpness of the nearfield intensity edge distribution. This results in an increase of the system energy utilization rate. Figure 27(a) represents the near-field distribution of the SG-II upgrade when operated at $8000 \mathrm{~J}$ and without the second binary mask. The edge is blurred, which means that the energy flux is low. In order to improve the steepness of the edge and to fully utilize the optics aperture to increase the output energy, the 2nd near-field binary mask was designed as shown in Figure 27(b). Figure 27(c) is the near-field distribution of the SG-II upgrade when operated at $17,600 \mathrm{~J}$ with the second near-field binary mask.

Though the binary mask can accurately define the nearfield intensity distribution, it cannot control this distribution in real time for long processing cycles. In that case, a spatial modulator is a good choice ${ }^{[59]}$. However, commercial spatial modulators are limited by their low-energy availability. Optically addressed spatial modulator can achieve high-energy availability without interferometric strips ${ }^{[60-62,64-67]}$.

\subsubsection{Optically addressed spatial modulator ${ }^{[60-62,64-67]}$}

As a transmission and amplitude type spatial light modulator, the optically addressable spatial light modulator has the advantages of high transmittance, high filling factor, no black gate and no diffraction spots in the follow-up optical path, compared with the existing TFT type transmission liquid crystal spatial light modulator. In high power laser systems, the use of modulators can optimize beam quality in real time. In particular, they can block the damage sites or hot spots over time, to enhance the life of the optical components and the operating system flux level of the laser system, thereby enhancing the system cost ratio ${ }^{[60-62]}$.

As shown in Figure 28, the optical guide layer is connected in series with a liquid crystal layer, and the partial pressure on this layer is adjusted by controlling the intensity of the address beam on the photoconductive layer. This controls the retardation of the liquid crystal layer. Subsequent deflector demodulation can achieve signal light (read beam) spatial intensity distribution control. 


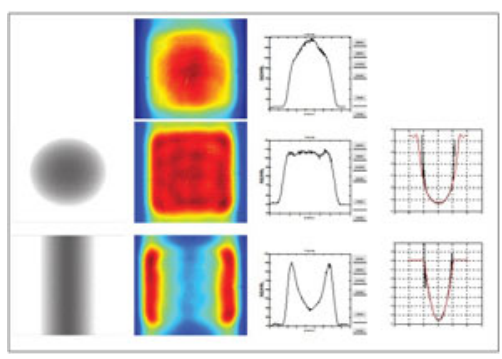

(a)

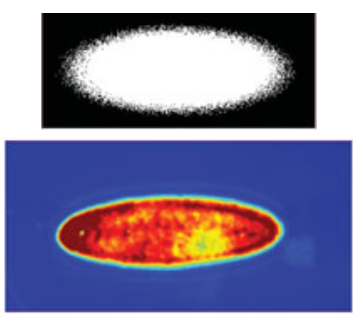

(b)

Figure 25. The experimental results of the uniform intensity distribution (a) after shaped by anti-Gauss beam mask and the parabola distribution after shaped by pre-compensating binary mask of which the peak/center transmission ratio is $5: 1$. (b) The elliptical near-field distribution using binary mask.

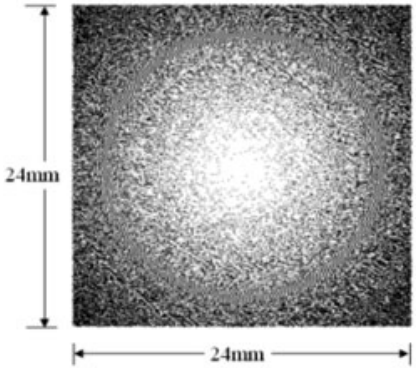

(a)

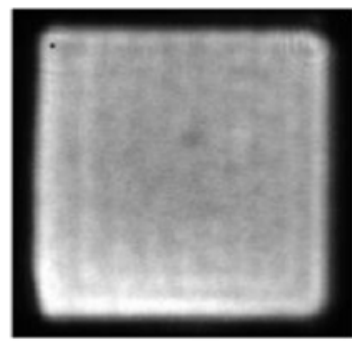

(b)

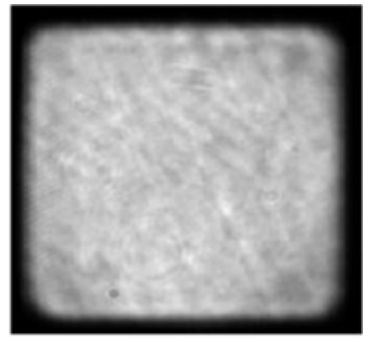

(c)

Figure 26. (a) The distribution of the binary mask. (b) The distribution of the four-pass amplifier without pre-compensation mask. (c) The output near-field distribution of the four-pass amplifier with pre-compensation mask.

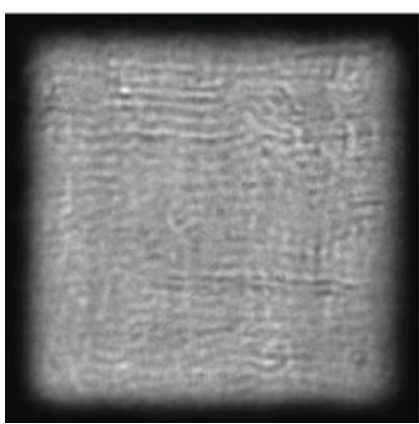

(a)

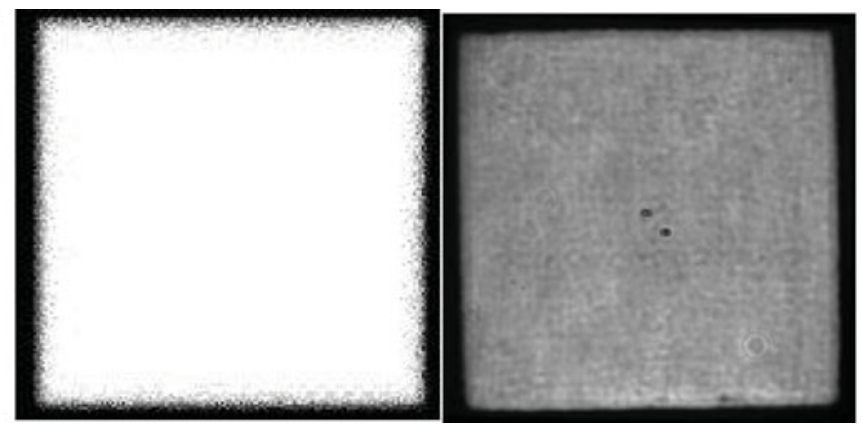

(b)

(c)

Figure 27. (a) The near-field distribution of SG-II-upgrade when operated at $8000 \mathrm{~J}$ without the second near-field binary shaping mask. (b) The design graphics of the 2 nd near-field binary shaping mask. (c) The near-field distribution of SG-II when operated at 17,600 J with the second near-field binary mask.

Through optimization of the liquid crystal mode, the liquid crystal box technology of the crystal substrate, the design and integrated design of the photosensitive optical imaging system, the following specifications of the optically addressed spatial modulator were achieved. These included a transmission rate of approximately $85 \%$ (for $1053 \mathrm{~nm}$ ), a wavefront deviation of $0.5 \lambda$ (for $\Phi 10 \mathrm{~mm}$ ), a damage threshold of $100 \mathrm{~mJ} / \mathrm{cm}^{2}$, an aperture of the optical valve of $22 \mathrm{~mm} \times 22 \mathrm{~mm}$ and an on-off ratio of $100: 1^{[67]}$.

The physical map of the optically addressed modulator is shown in Figure 29(a). The near-field spatial intensity distribution control results with the optically addressed spatial modulator are shown in Figure 29(b).
Optically addressed spatial modulators have been used in SG-II series facilities to accurately control the near-field intensity distribution in real time without affecting other parameters of the laser, such as the far field and laser spectrum $^{[67]}$. A high transmittance renders this kind of transmissive amplitude-mode spatial modulator very useful in many high power/energy laser systems.

Up to now, our laboratory has achieved a flexible nearfield intensity control technology, which facilitates both high damage threshold static near-field control components, and online real-time control of the transmission-type spatial light modulator technology. At present, the above two types of devices are used in the SG-II series device. As such, the 


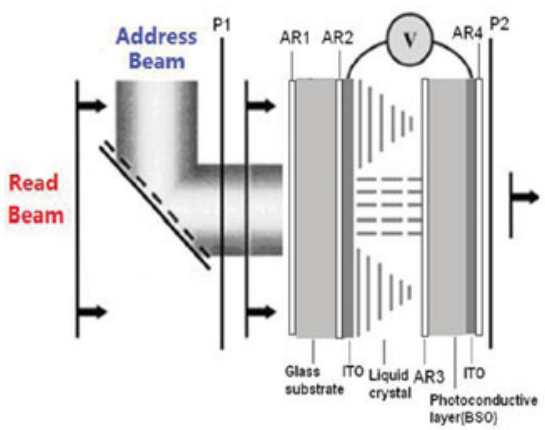

(a)

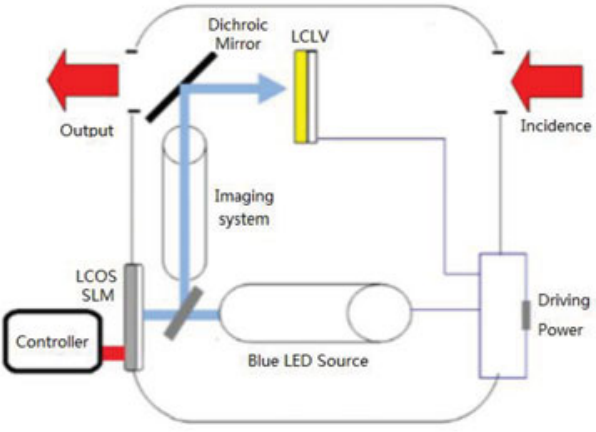

(b)

Figure 28. (a) Working principle and (b) the inner structure of the integrated optically addressed spatial modulator.

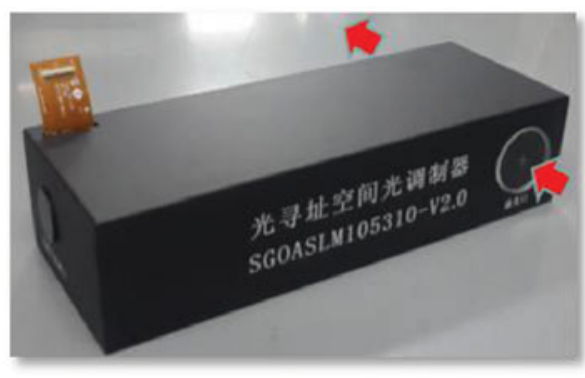

(a)
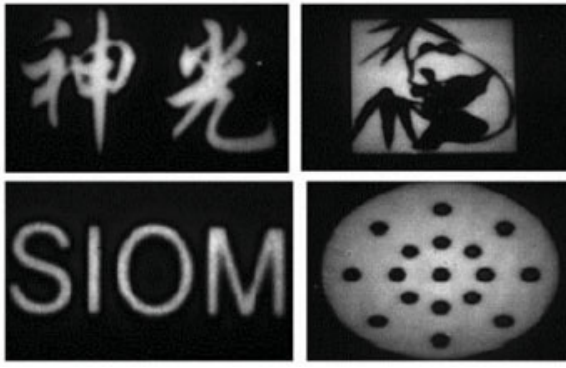

(b)

Figure 29. (a) Optical addressing liquid crystal spatial light modulator physical map. (b) Near-field spatial intensity distribution control demonstration.

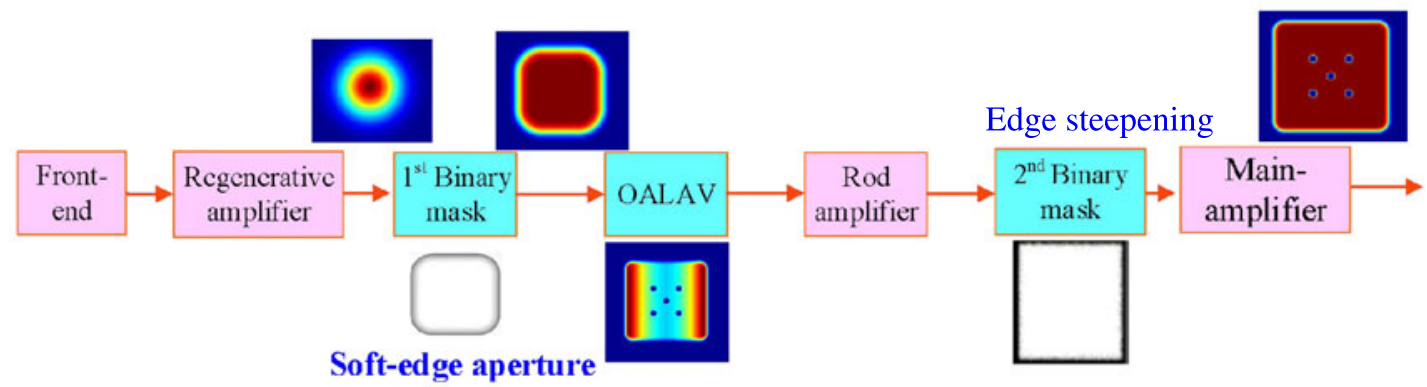

Figure 30. Near-field intensity distribution control strategy (OALAV: optically addressed liquid addressed valve).

following near-field intensity distribution control strategy (Figure 30) has been formed.

The first near-field control component is a binary mask. It is used to produce the soft-edge aperture to avoid Fresnel diffraction and to define the laser beam profile. The second component is an optically addressed spatial modulator, which is used to pre-compensate the spatial dependent gain of the amplifiers. It is also used to pre-block the hot spot of the laser or the damage sites of the large aperture optics. The third component is also a high damage binary mask, which is used to sharpen the near-field intensity edge distribution to improve the system energy utilization and the entire output energy.
In fact, because the size of the filter holes of the spatial filter influences the near-field intensity control resolution, high resistance to damage and flexible near-field spatial intensity distribution control components are needed. We are currently developing high damage threshold liquid crystal spatial light modulators in order to achieve more flexible near-field intensity control.

\subsection{Pre-amplifier technology}

4.4.1. High gain and high stability $\mathrm{Nd}^{3+}$ : glass regenerative amplifier

To amplify the nanojoule laser to a higher level, we previously adopted a $\mathrm{Nd}^{3+}$ :YLF double-stage amplifier for 


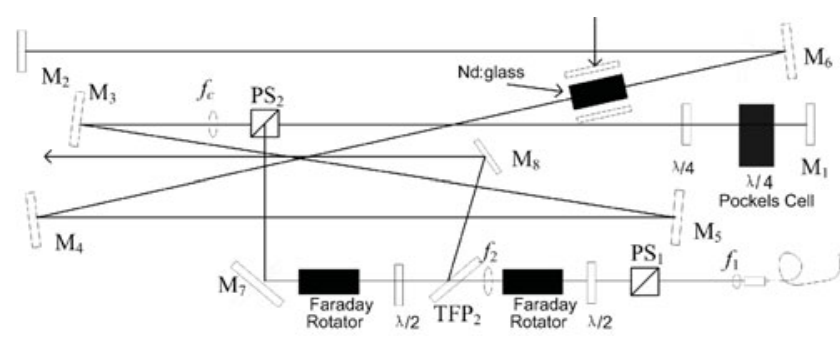

Figure 31. The scheme of the regenerative amplifier.

amplification up to $10 \mu \mathrm{J}$ for the eight beams of the SGII as shown in Figure 2. However, this approach has two major disadvantages. Firstly, it is a linear amplification so the output stability depends on the stability of the injection laser and $\mathrm{Nd}^{3+}$ :YLF amplifier. Secondly, the gain spectrum span of the $\mathrm{Nd}^{3+}: \mathrm{YLF}$ is about $0.1 \mathrm{~nm}$, which would induce FM-to-AM effect due to the gain narrowing. To resolve these problems, the $\mathrm{Nd}^{3+}$ :glass regenerative amplifier was developed $^{[72,73]}$.

This amplifier has three advantages which include high gain, high stability and wide spectrum amplification. A high stability is beneficial to maintaining the power balance of the high power laser system. The wide spectrum amplification supports the beam smoothing technologies for the focal spot.

A regenerative amplifier consists of an optical cavity with a gain medium, into which a low-energy seed optical pulse is injected, amplified by several orders of magnitude, and then switched out of the cavity ${ }^{[72,73]}$.

The configuration of a $\mathrm{Nd}^{3+}$ :glass regenerative amplifier is shown in Figure 31 where the cavity length determines the amplified laser pulse width. The crystal of the Pockels cell is $\mathrm{KD}^{*} \mathrm{P}$, which has a damage threshold of $850 \mathrm{MW} / \mathrm{cm}^{2}$ for a nanosecond laser. In order to avoid destroying the crystal when the regenerative amplifier operates in a saturation state, the oscillation adopts an asymmetric stable cavity. $\mathrm{The} \mathrm{Nd}^{3+}$ :glass crystal rod is placed at a specified distance away from the end mirror M2 to keep the injected pulse from overlapping in the $\mathrm{Nd}^{3+}$ :glass crystal rod, which may produce irregular pulse-shape distortion. The $\mathrm{Nd}^{3+}$ :glass crystal rod $(\Phi 4 \times 60 \mathrm{~mm})$ is side pumped by a $6 \mathrm{~kW} \mathrm{LD}$ array operating at $808 \mathrm{~nm}$ with a repetition rate of $1 \mathrm{~Hz}$ and an input pulse energy of $500 \mathrm{pJ}$. After 33 round trips in the cavity, the output energy is maximized to $10 \mathrm{~mJ}(5 \mathrm{~ns})$. The stability of the output energy is better than $0.3 \%$ (rms) shown in Figure 32(c), which was tested for $\sim 8$ hours. The square-pulse distortion (SPD) is less than $1.3: 1$ as shown in Figure 32(d) and the maximum amplified pulse width is 25 ns.

The high gain and high stability $\mathrm{Nd}^{3+}$ : glass regenerative amplifier has been in operation in the SG-II series facility for about 7 years.

\subsubsection{Single-shot $10 \mathrm{~J}$ pre-amplifier}

The traditional pre-amplifier system uses a single-shot technical solution; the goal is to provide an appropriate energy,

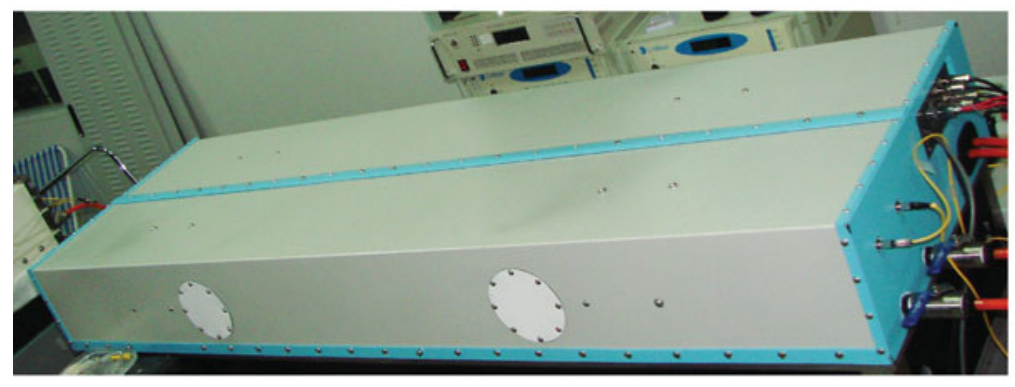

(a)

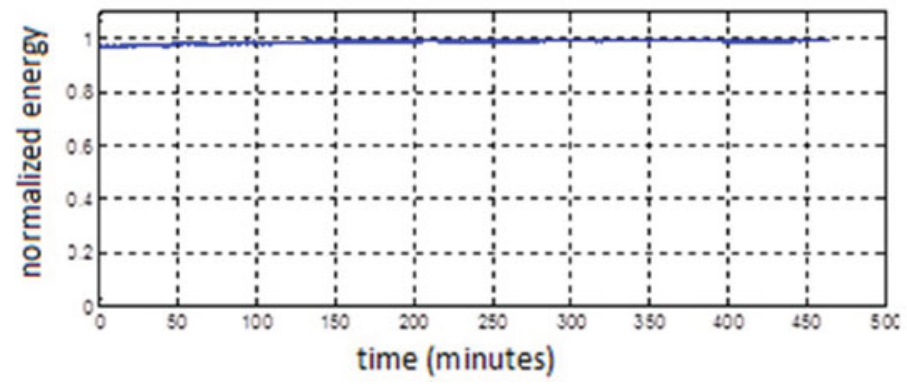

(c)

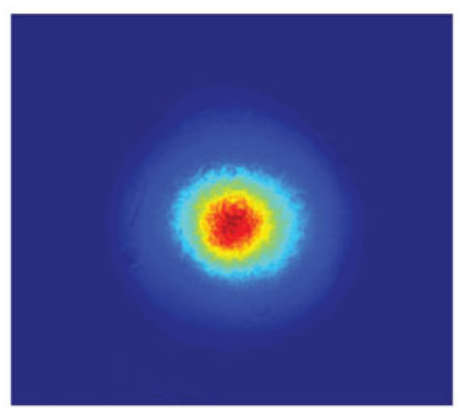

(b)

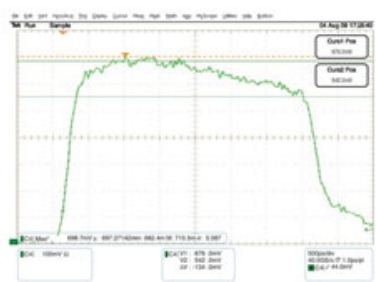

(d)

Figure 32. (a) The regenerative amplifier; (b) the output near-field spot; (c) the energy stability of the regenerative amplifier for one day; (d) the square-pulse distortion of the regenerative amplifier. 


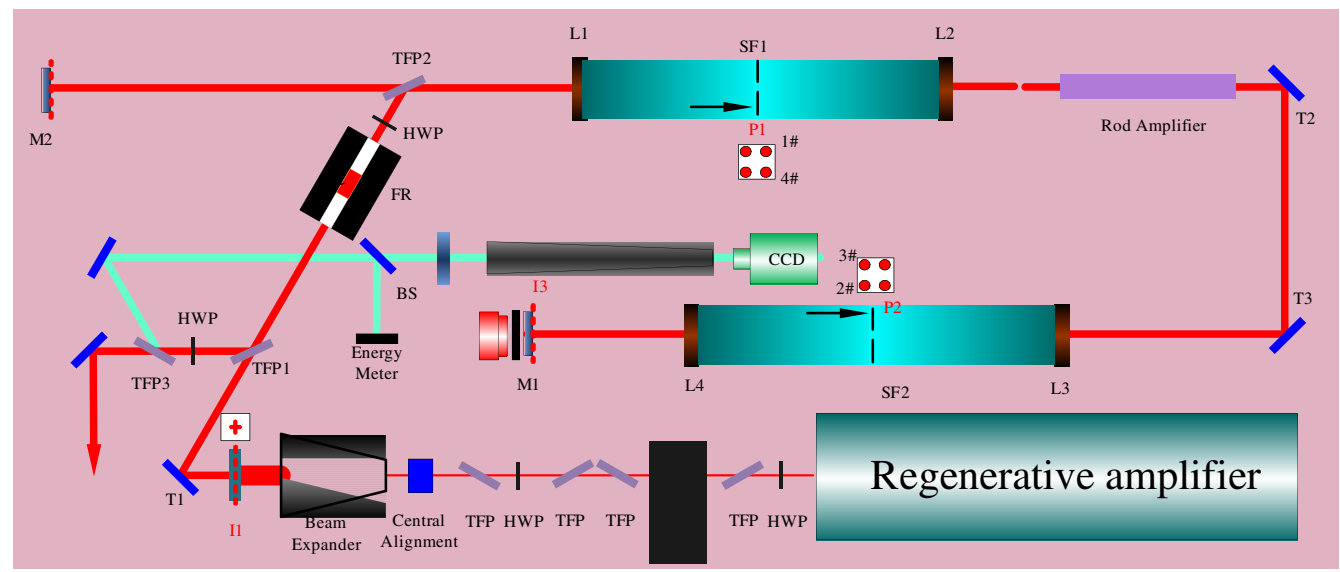

Figure 33. Off-axis four-pass amplifier optical path diagram.

the time waveform, and the appropriate near-field beam distribution of laser pulses. The pre-amplifier system's program is gradually transitioning from an earlier single-pass cascade amplification regime to a new multi-pass configuration represented by the NIF: regenerative amplifier + spatial shaping + off-axis quad-pass amplifier ${ }^{[10]}$.

The four-pass amplifier has a simple structure, reliable performance, and small size compared to the multi-stage amplifier. We have completed the prototype development of an off-axis four-pass amplifier as shown in Figure 33. The key difficulty involves weakening or even suppressing the parasitic oscillations of the four-pass amplifiers.

The total gain is 12,500 with a $0.8 \mathrm{~mJ}$ injection laser pulse energy. The near-field distribution of the output beam is square flattened as shown in Figure 34. A spot size of $18 \mathrm{~mm} \times 18 \mathrm{~mm}$ (1\% light intensity) was achieved and the contrast is $\sim 8 \%$. The near-field filling factor is $\sim 70 \%$. There is no obvious pencil beam spot in the near-field distribution, indicating that the pencil beam was suppressed. The farfield distribution is shown in Figure 35, which is 2 times the diffraction limit ${ }^{[74]}$.

4.4.3. Repetitive frequency joule neodymium glass amplifier In high power laser devices, real-time monitoring of time waveforms, spectra, etc. is critical due to the laser beam control of the injection laser system. The output energy of the pre-amplifier section in the case of repetition will effectively improve the efficiency of the system's automatic collimation and target aiming.

The key technologies of the repetitive frequency joule neodymium glass amplifier include: an appropriate gain medium ${ }^{[75]}$, gain uniformity control, laser head thermal lens effect and thermal backoff effect suppression and compensation, output beam quality control, suppression of ghost image point and self-oscillation within the cavity and so on.

Since the neodymium glass gain medium emission crosssectional area is small, the single-pass small signal gain is low. The use of appropriate multi-pass amplification configuration can reduce the requirements of a single-pass small signal gain.

Based on the transmittance of the spatial shaping unit and the basic output capacity of the regenerative amplifier, which is $10 \mathrm{~mJ}$, the four-pass amplifier only needs to achieve $30 \mathrm{~dB}$ gain. This would greatly reduce the system's design difficulty and thermal effects. The joule-class repetition neodymium glass pre-amplifier system uses a 'regenerative amplification + near-field intensity distribution control + four-pass amplification' as the basic technical configuration. A $1 \mathrm{~J} @ 1 \mathrm{~Hz}$ neodymium glass amplifier using a coaxial four-pass amplifier is shown in Figure 36.

The amplifier achieved a $1 \mathrm{~Hz}$ operation with a $1.2 \mathrm{~J}$ output capacity. The main technical indicators include the following ${ }^{[76]}$.

(1) Near-field intensity distribution

The beam size is $7 \mathrm{~mm} \times 7 \mathrm{~mm}$, and the near-field modulation is 1.2 as shown in Figure 37, which is achieved by near-field intensity distribution pre-compensation with an optically addressed liquid crystal light modulator.

(2) Far-field distribution

In Figure 38, the dotted line represents the far-field energy distribution of the 6th order super-Gaussian square spot, and the solid line represents the actual measurement result of the amplifier. A total of $95 \%$ of the energy is concentrated in $720 \mu \mathrm{rad}$, which is 2.3 times the diffraction limit.

(3) Output energy and the stability

The output energy is $1.2 \mathrm{~J}$; the stability is $6.6 \%$ (PV) and 1.2 (rms) for 30 minutes as shown in Figure 39.

\subsection{High signal-to-noise ratio picosecond pulsed light source technology}

The OPCPA has the advantages of wide gain bandwidth, high-energy conversion efficiency, good beam quality, short 


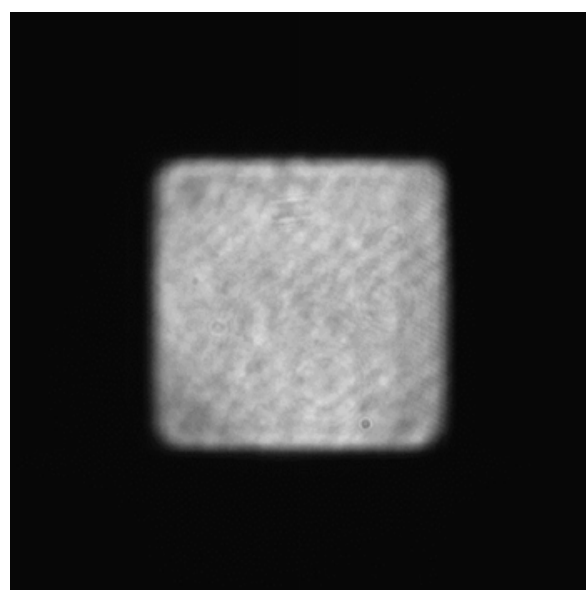

(a)

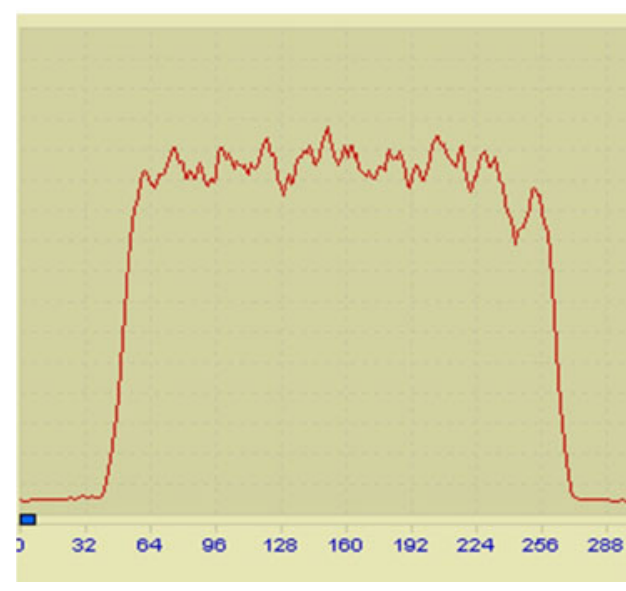

(b)

Figure 34. Four-pass amplifier near-field beam spatial distribution. (b) is the one-dimensional distribution of (a).

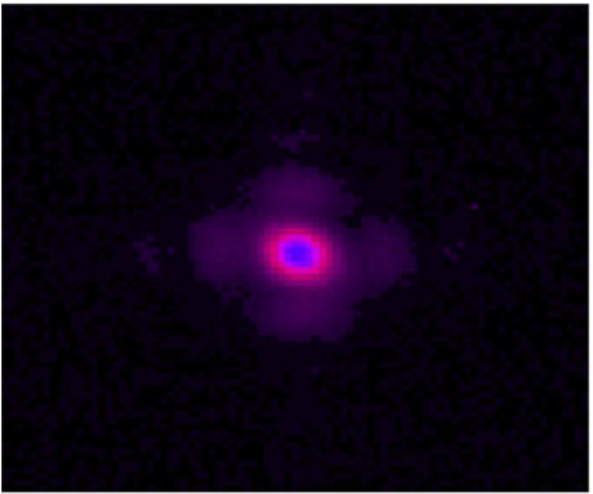

(a)

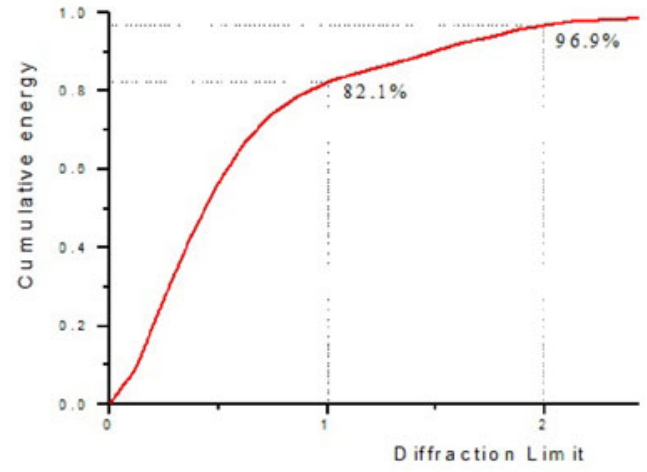

(b)

Figure 35. Four-pass pre-amplifier output focal spot distribution. (a) Two-dimensional distribution; (b) surrounding energy distribution.

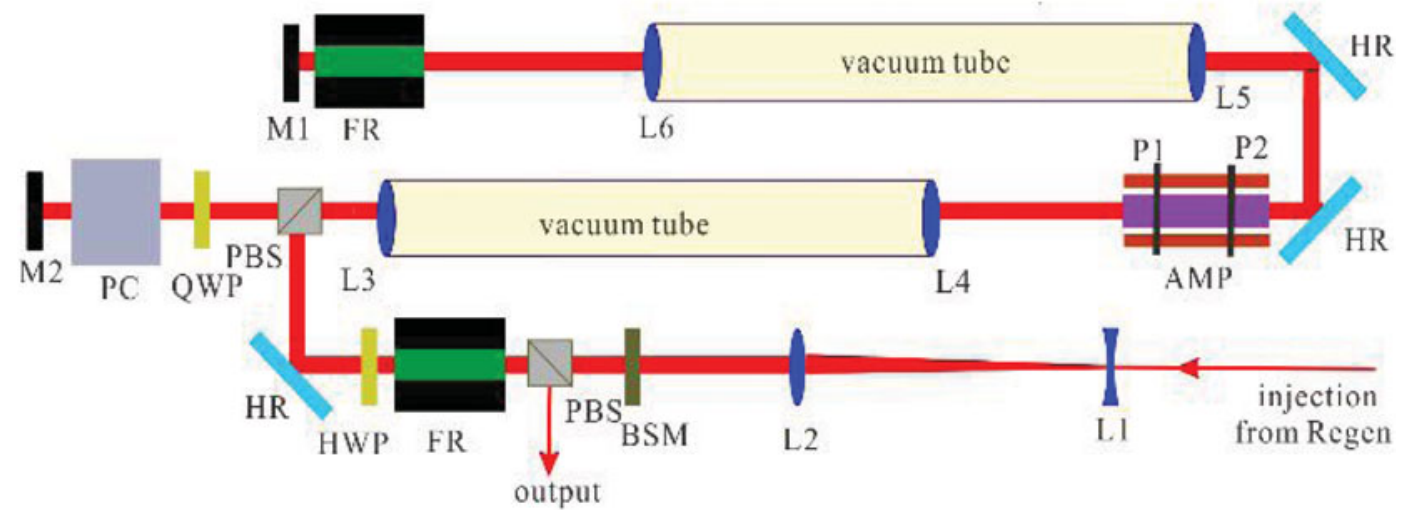

Figure 36. Coaxial four-pass amplifier structure.

working medium, low heat deposition, low phase distortion and high signal-to-noise ratio. It is the key front-end technology for the future development of high-energy PW laser systems and EW $\left(10^{18} \mathrm{~W}\right)$ ultra-high power laser systems $^{[77,78]}$. The high gain, high signal-to-noise ratio picosecond OPCPA seed source was developed to improve 


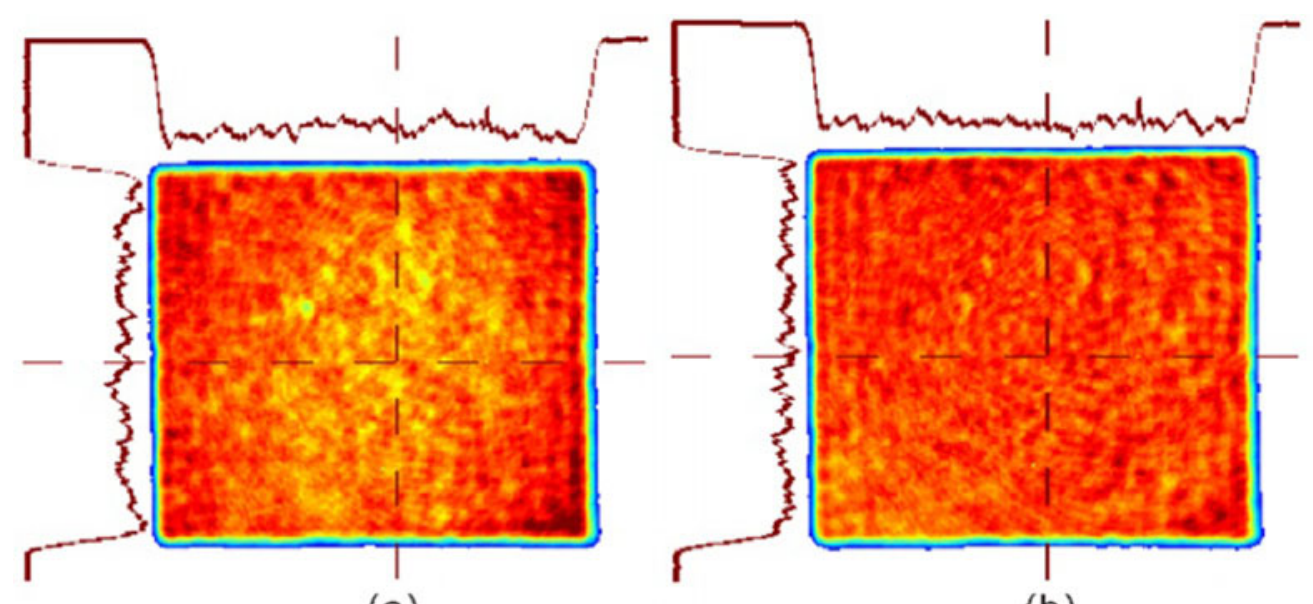

(a)

(b)

Figure 37. Near-field intensity distribution (a) without pre-compensation and (b) with pre-compensation.

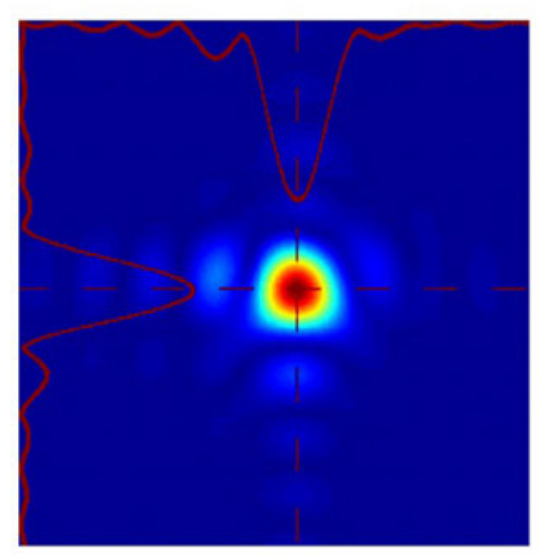

(a)

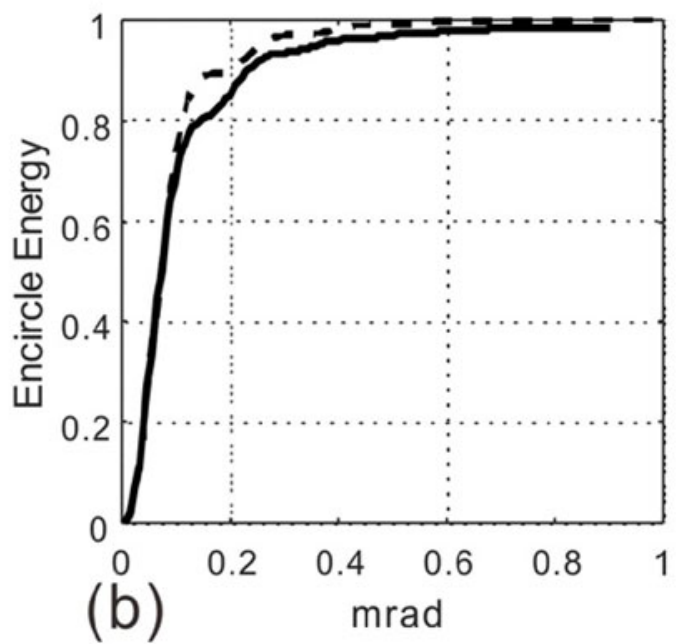

(b) mrad

Figure 38. (a) Far-field intensity distribution and (b) surrounding energy distribution.

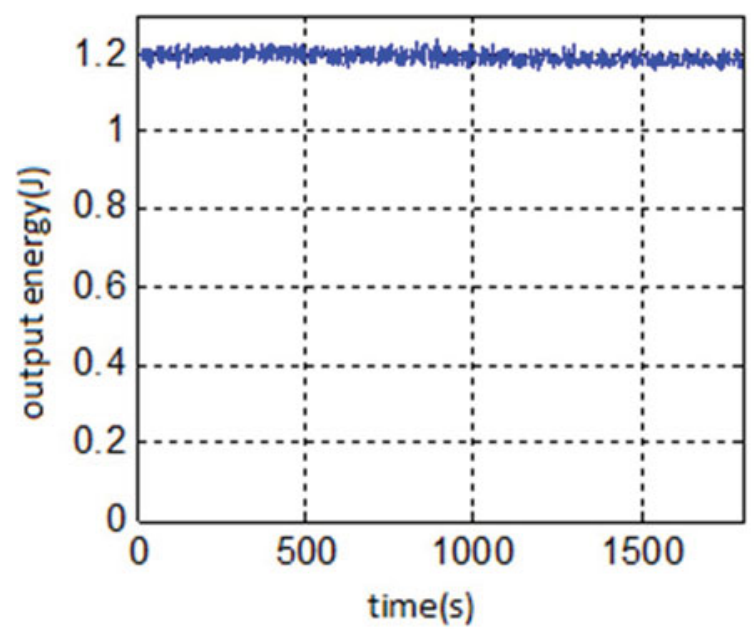

Figure 39. The output energy stability of repetition pre-amplifier.

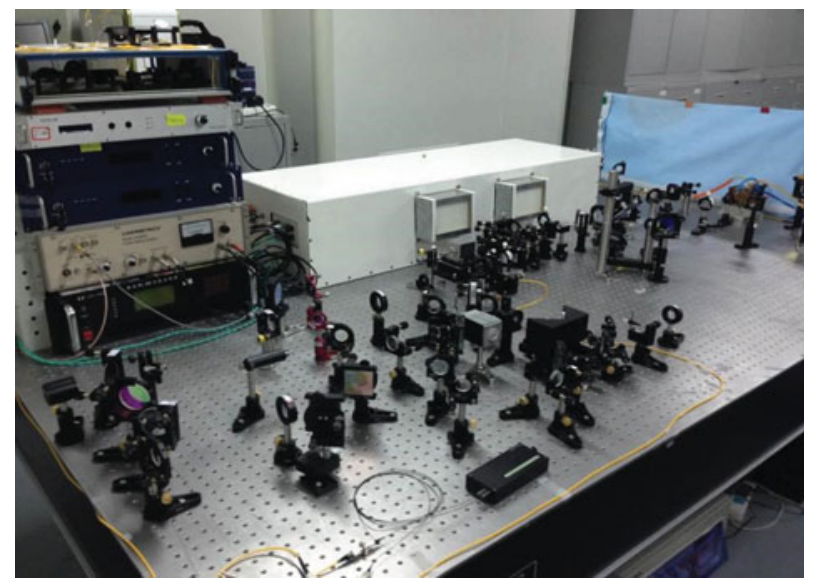

Figure 40. Picosecond joule multi-functional experimental platform. 


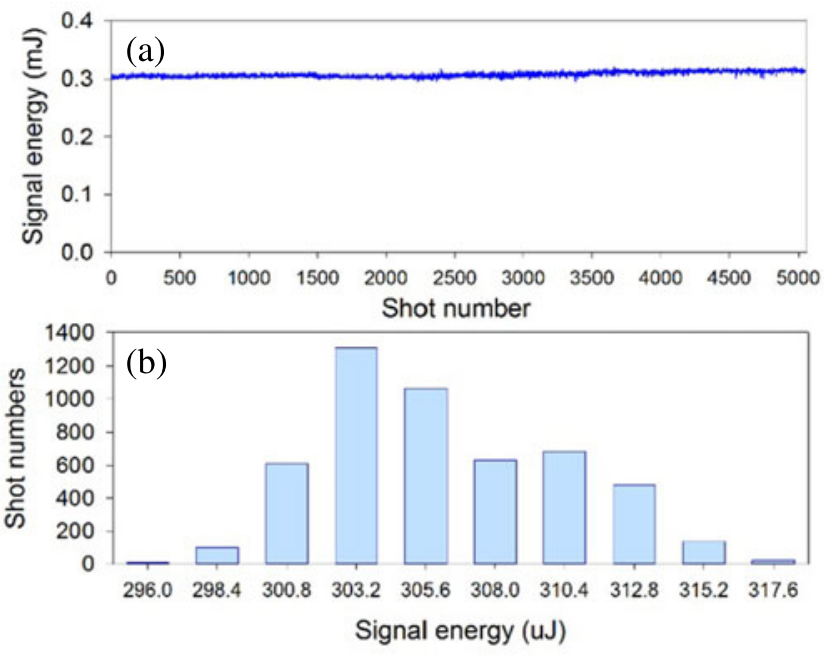

Figure 41. (a) Output energy changes and (b) stability histograms.

the signal-to-noise ratio of the entire high power laser system. It was considered from the perspective of the system's noise, which limited the parametric fluorescence to the picosecond time domain window. Simultaneously, the gain of the following nanosecond field OPCPA is decreased, which can reduce the noise intensity in the nanosecond window. This is currently one of the main ways to improve the signal-to-noise ratio.

The high-gain high signal-to-noise ratio picosecond OPCPA seed source achieves $10^{8}$ high signal-to-noise ratio under $300 \mu \mathrm{J}$ output energy condition, and on this basis, the picosecond joule multi-function experimental platform was realized as shown in Figure 40 ${ }^{[79]}$.

Its main technical indicators include the following.

(1) Output energy and stability

The output energy of parametric amplifier is $307 \mu \mathrm{J}$, the energy stability is $1.35 \%$ (rms) for 5000 shots as shown in Figure 41.

(2) Output spectrum and compressed pulse

The full width at half-maximum of the output spectrum of the injection and the output of the parametric amplifier is $6 \mathrm{~nm}$ (Figure 42(a)). The pulse width is $414 \mathrm{fs}$ (Figure 42(b)) after the grating compressing, which basically reached the Fourier transform limit.

(3) Signal-to-noise ratio

The signal-to-noise ratio at $200 \mathrm{ps}$ before the main pulse is shown in Figure 43, and the signal-to-noise ratio beyond $20 \mathrm{ps}$ of the main pulse reached $10^{8}$.

\section{(4) Beam quality}

The magnification of the output light is $3 \mathrm{~mm}$, the nearfield light spot is shown in Figure 44(a), the near-field wavefront is $0.25 \lambda$ (Figure 44(b)), a total energy of $87 \%$ is concentrated within $1 \mathrm{mrad}$, and the Strehl factor is 0.8 (Figure 44(c)).

\subsection{Nanosecond joule OPCPA pump source technology}

In order to obtain better OPCPA output stability, a highquality OPCPA pump source requires a square-pulse waveform, a flat-top near-field distribution, and a stable output energy, and the synchronization between the seed source and the pump source should be accurate. We constructed a set of high stability joule-class OPCPA pump sources with a single-longitudinal-mode laser and waveguide modulator pulse shaping system, an LD pumped Nd:YAG regenerative amplifier, a binary mask for near-field control, a xenon lamppumped Nd:YAG traveling wave amplifier and frequency doubling. The nanosecond OPCPA pump source setup is shown in Figure 45. It has been put into operation since 2010. The output time waveform of the front end and the final

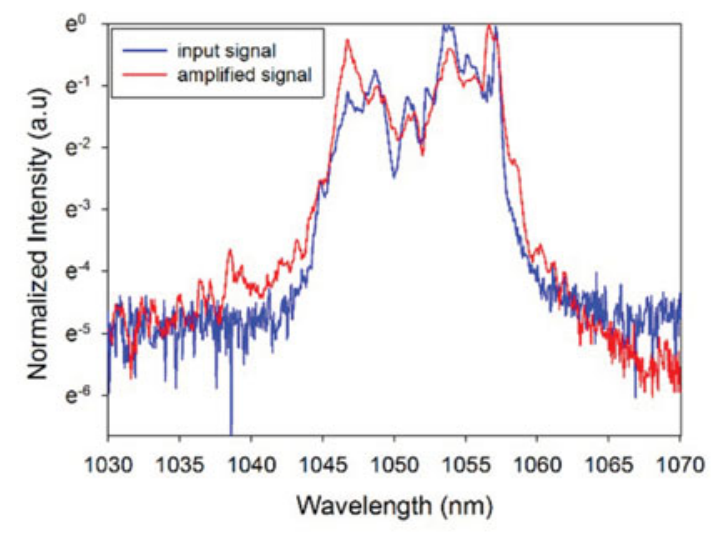

(a)

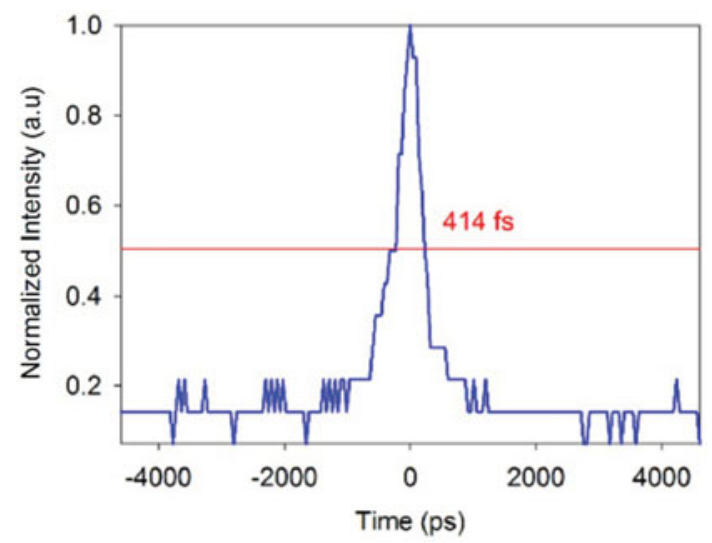

(b)

Figure 42. (a) Injection and output spectra. (b) Pulse width after compression. 


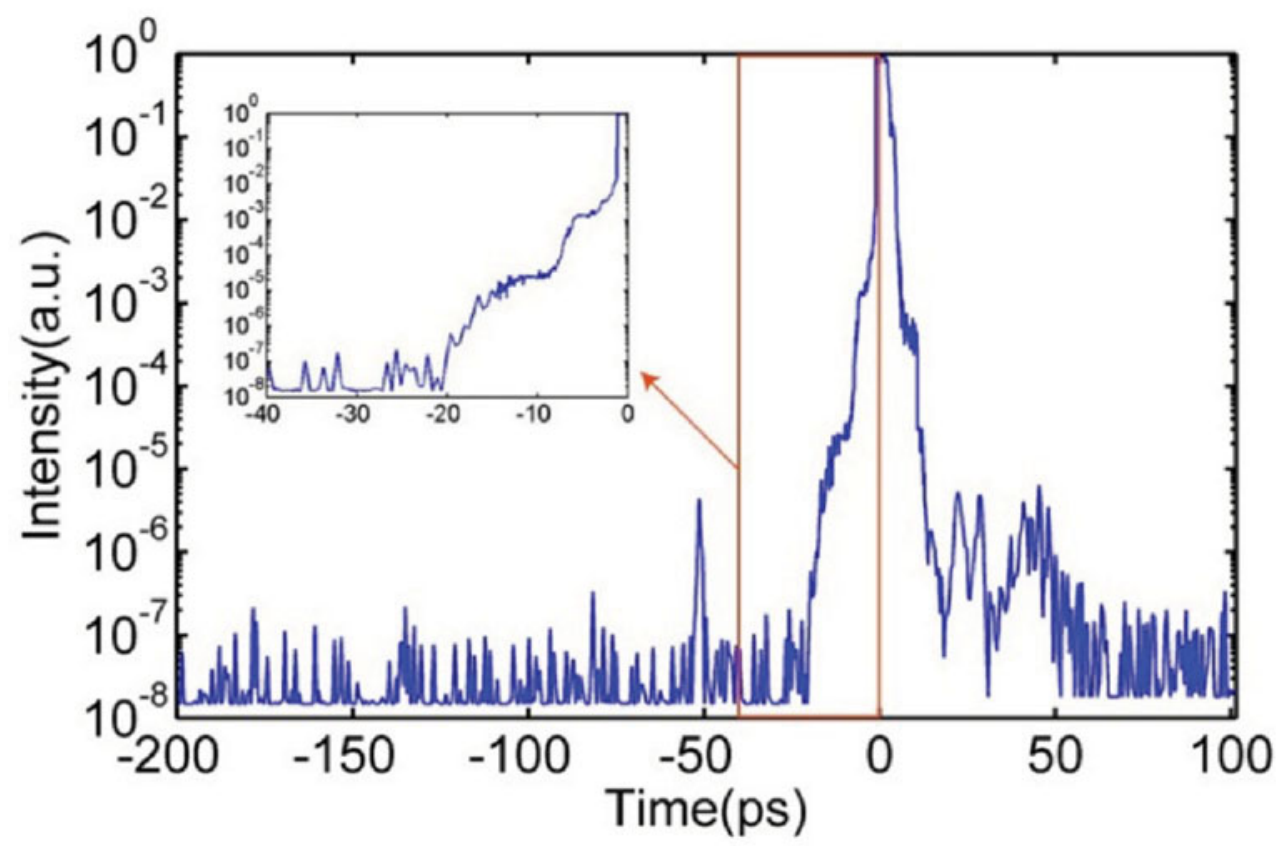

Figure 43. Parameter to amplify the signal-to-noise ratio measurement after compression.

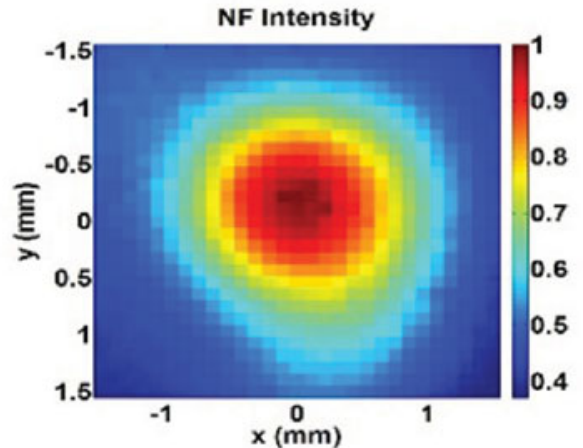

(a)

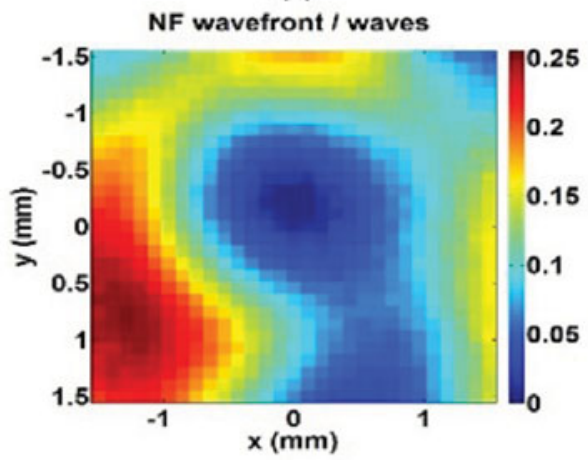

(b)

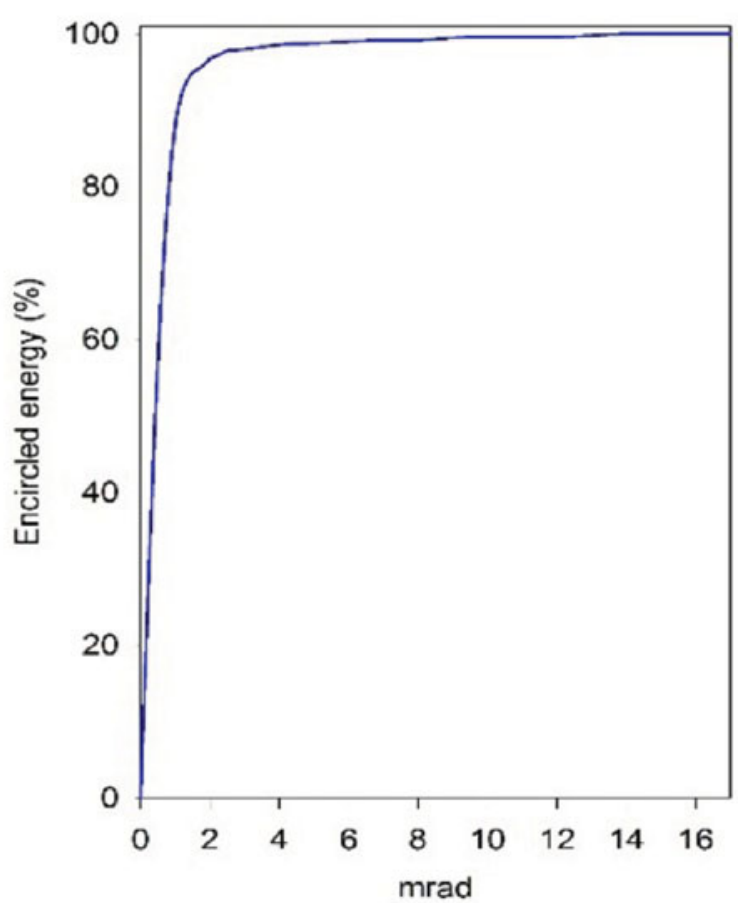

(c)

Figure 44. (a) The near-field light spot. (b) The near-field wavefront. (c) Far-field ambient energy. 




Figure 45. The OPCPA pump source includes: Nd:YAG regenerative amplifier, beam expander, soft-edge iris, spatial filter, three-stage Nd:YAG rod amplifier and frequency multiplier.

output of the OPCPA pump source are shown in Figure 46. The energy stability of the OPCPA pump source is shown in Figure 47 and the near-field distribution is shown in Figure 48.

The main technical indicators are as follows:

(1) output energy (@532 nm): $\geqslant 1 \mathrm{~J}$;

(2) output energy stability: 1\% (rms);

(3) synchronization accuracy: $\leqslant 5$ ps (rms);

(4) output wavelength: $532 \mathrm{~nm}$, single-longitudinal mode;

(5) waveform: $\sim 8$ ns square wave;

(6) beam diameter: $\sim 10 \mathrm{~mm}$;

(7) near-field light intensity spatial distribution: approximately flat;

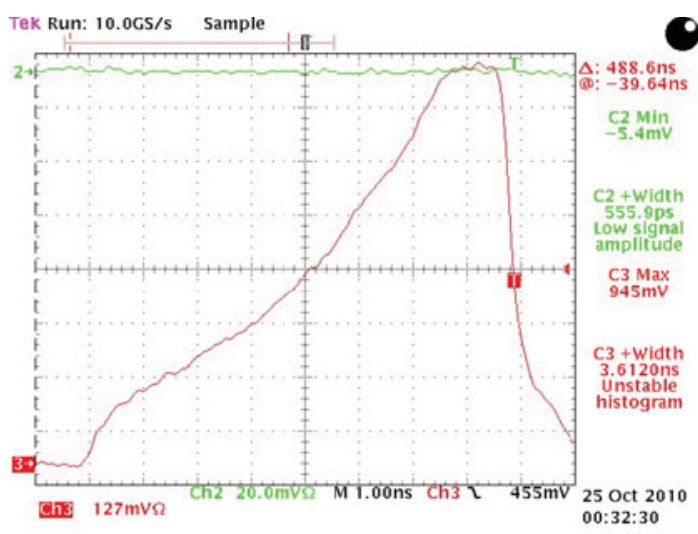

(a)
(8) repetitive operating frequency: $1 \mathrm{~Hz}$.

The highly stable joule-level OPCPA pump source is a key technological platform for the OPCPA high-performance output.

\section{Summary}

The injection laser system is the source for the entire high power laser system. The control ability of the injection laser system determines the control ability of the laser system to some extent. Although the injection laser system of the SGII series can control the laser in the time domain, spatial domain, and the frequency domain, temporal computer control and feedback control require further work.

Ignition has not been achieved yet. It is known that the uniformity of the focal spot will influence the capsule's compression effect. In order to improve the focal spot smoothness, smoothing by spectral dispersion was adopted. The smoothing time should ideally be less than the interaction time of the laser and plasma to avoid nonlinear effects. However, the smoothing time depends on the bandwidth of the phase modulator. That means that if the interaction time of the laser and plasma is about $10 \mathrm{ps,} \mathrm{the} \mathrm{bandwidth}$ of the phase modulator should be greater than $100 \mathrm{GHz}$, which cannot be achieved using current technology. In addition, the wider the bandwidth of the phase modulator, the wider the broadened spectrum, which must be considered for uniform amplification. Another concept involves spike trains of uneven delay and duration (STUD) pulses and was investigated at Trident. In this approach, the laser on and off time is ps-scaled, which may be less than the relaxation time of the LPI ${ }^{[80,81]}$. STUD pulses may minimize the long-term accumulation of parametric growth and plasma disruption. Therefore, the issue of controlling the laser characteristics to decrease the LPI and acquire implosion symmetry is the key problem which must be addressed for current injection laser systems of ICF high power laser drivers.

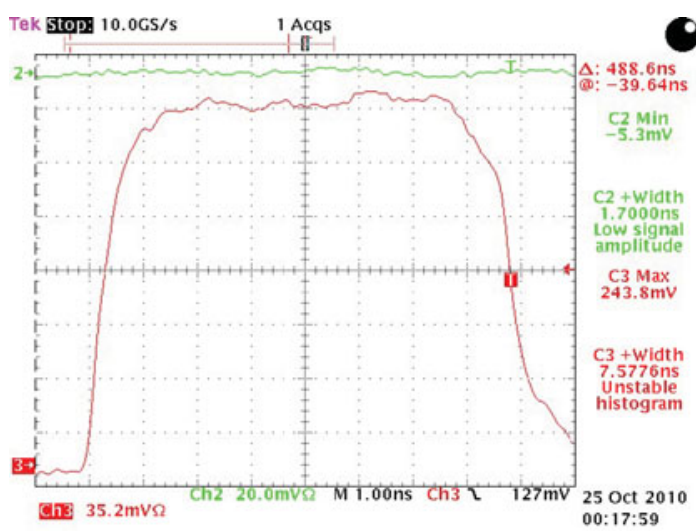

(b)

Figure 46. (a) The output time waveform of the front end and (b) final output of the OPCPA pump source. 


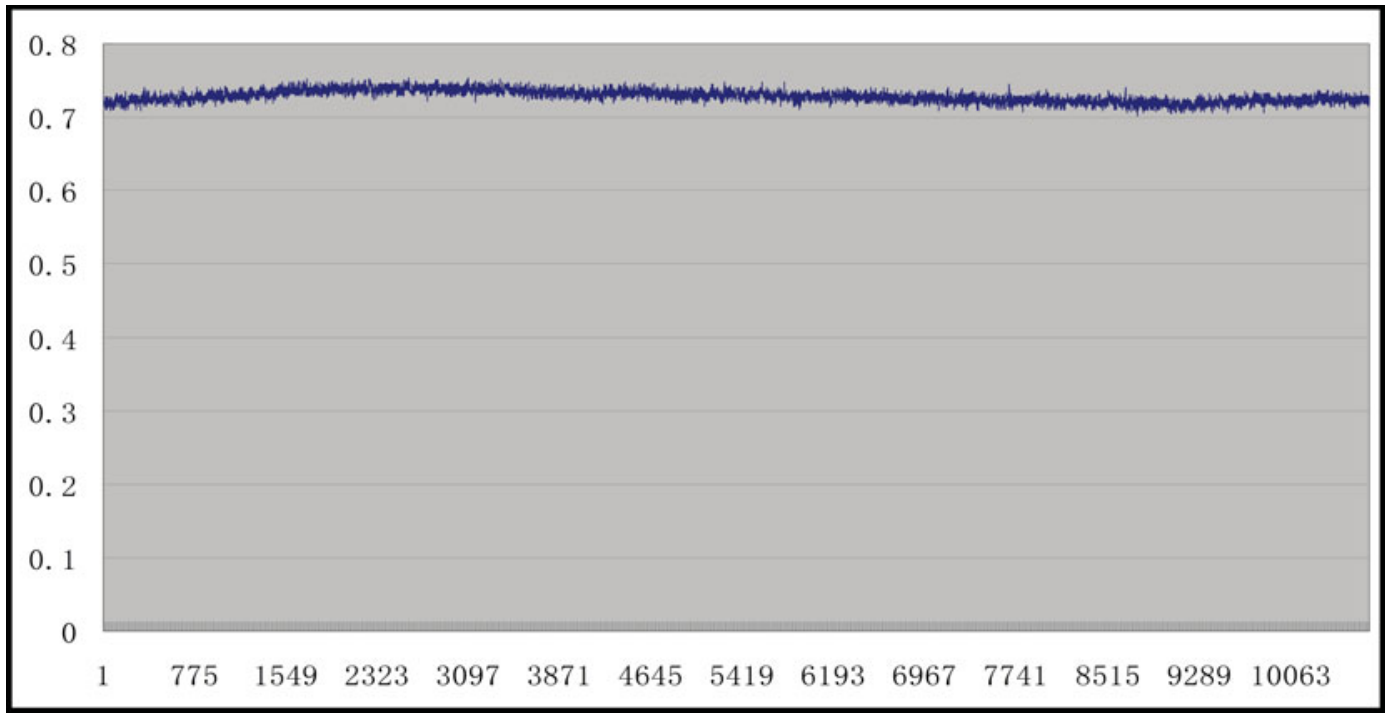

Figure 47. The energy stability of the OPCPA pump source.

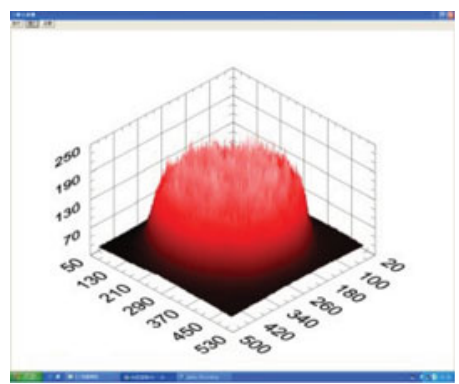

(a)

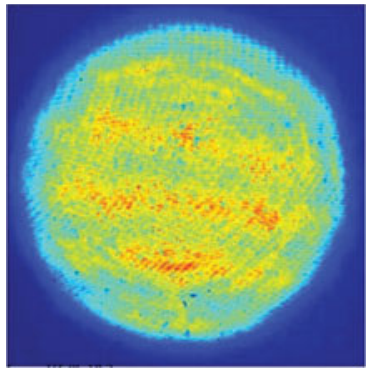

(b)

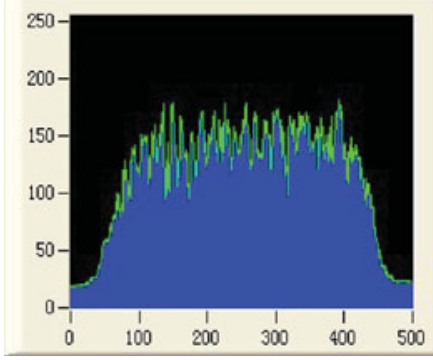

(c)

Figure 48. The near-field distribution of the OPCPA pump source.

\section{References}

1. M. L. Spaeth, K. R. Manes, D. H. Kalantar, P. E. Miller, J. E. Heebner, E. S. Bliss, D. R. Speck, T. G. Parham, P. K. Whitman, P. J. Wegner, P. A. Baisden, J. A. Menapace, M. W. Bowers, S. J. Cohen, T. I. Suratwala, J. M. Di Nicola, M. A. Newton, J. J. Adams, J. B. Trenholme, R. G. Finucane, R. E. Bonanno, D. C. Rardin, P. A. Arnold, S. N. Dixit, G. V. Erbert, A. C. Erlandson, J. E. Fair, E. Feigenbaum, W. H. Gourdin, R. A. Hawley, J. Honig, R. K. House, K. S. Jancaitis, K. N. LaFortune, D. W. Larson, B. J. Le Galloudec, J. D. Lindl, B. J. MacGowan, C. D. Marshall, K. P. McCandless, R. W. McCracken, R. C. Montesanti, E. I. Moses, M. C. Nostrand, J. A. Pryatel, V. S. Roberts, S. B. Rodriguez, A. W. Rowe, R. A. Sacks, J. T. Salmon, M. J. Shaw, S. Sommer, C. J. Stolz, G. L. Tietbohl, C. C. Widmayer, and R. Zacharias, Fusion Sci. Technol. 69, 25 (2016).

2. LLE Review Quanterly Report, University of Rochester Laboratory for Laser Energetics (1998), DOE/SF/19460-264.

3. C. Cavailler, N. A. Fleurot, and J. M. DiNicola, Proc. SPIE 5580, 443 (2005).

4. W. G. Zheng, X. Wei, Q. Zhu, F. Jing, D. X. Hu, J. Q. Su, K. X. Zheng, X. D. Yuan, H. Zhou, W. J. Dai, W. Zhou, F. Wang, D. P. Xu, X. D. Xie, B. Feng, Z. T. Peng, L. F. Guo, Y. B. Chen,
X. J. Zhang, L. Q. Liu, D. H. Lin, Z. Dang, Y. Xiang, and X. W. Deng, High Power Laser Sci. Eng. 4, e21 (2016).

5. J. Q. Zhu, Bull. Chin. Acad. Sci. 20, 42 (2005).

6. D. Pile, Nat. Photon. 11, 644 (2011).

7. X. Liu, Y. Y. Li, Y. Zhang, J. Y. Zhong, W. D. Zheng, Q. L. Dong, M. Chen, G. Zhao, Y. Sakawa, T. Morita, Y. Kuramitsu, T. N. Kato, L. M. Chen., X. Lu, J. L. Ma, W. M. Wang, Z. M. Sheng, H. Takabe, Y.-J. Rhee, Y. K. Ding, S. E. Jiang, S. Y. Liu, J. Q. Zuhu, and J. Zhang, New J. Phys. 13, 093001 (2011).

8. J. Y. Zhong, Y. T. Li, X. G. Wang, J. Q. Wang, Q. L. Dong, C. J. Xiao, S. J. Wang, X. Liu, L. Zhang, L. An, F. L. Wang, J. Q. Zhu, Y. Gu, X. T. He, G. Zhao, and J. Zhang, Nat. Phys. 6, 984 (2010).

9. P. J. Wisoff, M. W. Bowers, G. V. Erbert, D. F. Browning, and D. R. Jedlovec, Proc. SPIE 5341, 146 (2004).

10. M. Bowers, S. Burkhart, S. Cohen, G. Erbert, J. Heebner, M. Hermann, and D. Jedlovec, Proc. SPIE 6451, 64511M (2007).

11. X. C. Li, W. Fan, H. Wei, H. D. Zhu, J. F. Wang, J. Xie, L. Wang, J. L. Chen, B. Chen, S. J. Zhang, S. H. Chen, and Z. Q. Lin, Rev. Laser Eng. 2008, 1168 (2008).

12. W. L. Cao, Q. H. Chen, Z. M. Zhu, S. H. Chen, X. M. Deng, Y. L. Sun, and Q. Xie, Acta Opt. Sin. 6, 769 (1986). 
13. X. L. Xie, S. H. Chen, Z. Q. Lin, S. X. Meng, and Z. L. Ma, Laser Optoelectron. Prog. 36, 71 (1999).

14. S. H. Chen, Y. M. Chen, T. L. Chen, X. D. Shi, Y. Yang, and X. M. Deng, Chin. Sci. Bull. 36, 1542 (1991).

15. S. H. Chen, T. L. Chen, Y. M. Chen, X. D. Shi, Y. Yang, and X. M. Deng, Acta Opt. Sin. 11, 1091 (1991).

16. S. H. Chen, T. L. Chen, Y. M. Chen, G. P. Sheng, X. D. Shi, X. D. Guo, J. Q. Zhu, Y. Yang, and X. M. Deng, Sci. Chin. A 23, 611 (1993).

17. L. Shen, S. H. Chen, B. Y. Liu, S. Z. Xu, Z. L. Ma, H. Wei, and S. J. Zhang, Acta Opt. Sin. 23, 598 (2003).

18. W. Fan, X. T. Yang, X. C. Li, and H. D. Zhu, Opt. Laser Technol. 39, 1189 (2007).

19. B. Chen, W. Fan, X. C. Li, J. L. Chen, Q. Q. Qiao, S. H. Chen, and Z. Q. Lin, Chin. J. Lasers 29, 512 (2002).

20. Y. K. Gao, Y. T. Jiang, and X. C. Li, Chin. J. Lasers 32, 1619 (2005).

21. J. F. Wang, H. D. Zhu, X. C. Li, and J. Q. Zhu, Chin. J. Lasers 35, 31 (2008).

22. S. Hocquet, D. Pennincks, E. Bordenave, C. Gouedard, and Y. Jaouen, Appl. Opt. 47, 3338 (2008).

23. H. Storoy, B. Sahlgren, and R. Stubbe, Electron. Lett. 33, 56 (1997).

24. D. Kyrazis and T. L. Wiland, Proc. SPIE 1441, 469 (1990).

25. S. F. Guo, Q. S. Lu, X. A. Cheng, G. M. Zhao, H. M. Jiang, P. Zhou, and S. Y. Deng, High Power Laser Particle Beams 15, 850 (2003)

26. J. F. Gleyze, J. H. S. Vidal, N. Beck, J. Dubertrand, and A. Perrin, Proc. SPIE 7916, 791601 (2007).

27. A. Jolly, J. F. Gleyze, J. Luce, H. Coic, and G. Deschaseaux, Opt. Eng. 42, 1427 (2003).

28. R. Zhang, J. Su, J. Wang, L. Liu, P. Li, F. Jing, X. Zhang, L. $\mathrm{Xu}$, and H. Ming, Appl. Opt. 50, 687 (2011).

29. R. Zhang, X. Zhang, Z. Sui, and H. Ming, Opt. Laser Technol. 43, 1073 (2011).

30. J. E. Rothenberg, Proc. SPIE 2633, 634 (1995).

31. Two-Dimensional SSD on OMEGA, LLE Review (1996).

32. B. E. Kruschwitz and J. H. Kelly, Proc. SPIE 8602, 86020E (2013).

33. LLE Review Quarterly Report 114, 73-80, Laboratory for Laser Energetics, University of Rechester, Rochester, NY, LLE Document No. DOE/NA/28302-826, OSTI ID 93524 (2008).

34. Z. Lin, A. L. Lei, F. Wei, Z. Shenlei, and W. Li, High Power Laser Sci. Eng. 1, 110 (2013).

35. Y. E. Jiang, X. C. Li, S. L. Zhou, W. Fan, and Z. Q. Lin, Chin. Opt. Lett. 11, 052301 (2013).

36. J. D. Zuegel and D. W. Jacobs-Perkins, Appl. Opt. 43, 1946 (2004).

37. Microwave phase modulators for smoothing by spectral dispersion, LLE Review (1996).

38. D. Kyrazis and T. L. Wiland, Proc. SPIE 1441, 469 (1990).

39. J. E. Rothenberg, D. F. Browning, and R. B. Wilcox, Proc. SPIE 3492, 51 (1999).

40. Y. Y. Zhang, G. Y. Li, W. Fan, X. C. Wang, Y. E. Jiang, and Z. D. Shi, Laser Optoelectron. Prog. 53, 081405 (2016).

41. The design of optical pulse shapes with an aperture-coupledstripline pulse-shaping system, LLE Review (2006).

42. K. Rambabu and J. Bornemann, in IEEE MTT-S International Microwave Symposium Digest (2002), p. 97.

43. S. C. Burkhart, R. J. Beach, J. H. Crane, J. M. Davin, M. D. Perry, and R. B. Wilcox, Proc. SPIE 2633, 48 (1995).

44. D. Li, B. Y. Liu, J. Y. Liu, X. Ouyang, Y. L. Bai, X. H. Bai, C. Wang, J. S. Tian, L. Huang, and H. Li, Acta Photon. Sin. 34, 1304 (2005).
45. L. J. Waxer and J. H. Kelly, Opt. Lett. 27, 1427 (2002).

46. D. P. Xu, R. Zhang, X. C. Tian, D. D. Zhou, N. Zhu, Z. Y. Zong, J. J. Wang, M. Z. Li, D. X. Hu, Q. H. Zhu, and X. M. Zhang, Laser Optoelectron. Prog. 54, 020005 (2017).

47. J. P. Armstrong, A. Bayramian, and R. Beach, in Advanced Solid-State Photonics (OSA, 2007), paper MB1.

48. https://lasers.llnl.gov/multimedia/publications/photons_fusion /2012/julyphp.

49. A. Jolly, J. F. Gleyze, D. Penninckx, N. Beck, L. Videau, and H. Coic, C. R. Phys. 7, 198 (2006).

50. D. Penninckx and N. Beck, Appl. Opt. 44, 7773 (2005).

51. D. Penninckx, N. Beck, J. F. Gleyze, and L. Videau, J. Lightwave Technol. 24, 4197 (2006).

52. S. Vidal, J. Luce, and D. Penninckx, Opt. Lett. 36, 88 (2011).

53. http://lasers.1lnl.gov/news/science-technology/2016/october.

54. Q. Zhi, X. C. Wang, W. Fan, X. C. Li, Y. E. Jiang, R. Li, C. H. Huang, and Z. Q. Lin, Appl. Opt. 55, 8352 (2016).

55. C. H. Huang, X. H. Lu, Y. E. Jiang, X. C. Wang, Z. Qiao, and W. Fan, Appl. Opt. 56, 1610 (2017).

56. C. Dorrer and J. D. Zuegel, J. Opt. Soc. Am. B 24, 1268 (2007).

57. https://lasers.llnl.gov/news/photons-fusion/2012/june.

58. T. Bontoux, T. Saiki, T. Kanabe, H. Fujita, and M. Nakatsuka, Opt. Rev. 5, 234 (1998).

59. H. X. Chen, Z. Sui, Z. P. Chen, B. An, and M. Z. Li, Acta Opt. Sin. 21, 1107 (2001).

60. A. A. S. Awwal, R. Leach, G. Brunton, E. Tse, J. Matone, and J. Heebner, LLNL-CONF-464231(December 20, 2010).

61. https://www.rdmag.com/article/2012/06/2012-r-d-100-award -winners.

62. J. Heebner, M. Borden, P. Miller, C. Stolz, T. Suratwala, P. Wegner, M. Hermann, M. Henesian, C. Haynam, S. Hunter, K. Christensen, N. Wong, L. Seppala, G. Brunton, E. Tse, A. Awwal, M. Franks, E. Marley, K. Williams, M. Scanlan, T. Budge, M. Monticelli, D. Walmer, S. Dixit, C. Widmayer, J. Wolfe, J. Bude, K. McCarty, and J. M. DiNicola, LLNLPROC-462911 (November 22, 2010).

63. X. Jie, W. Fan, X. C. Li, and Z. Q. Lin, Acta Opt. Sin. 28, 1959 (2008).

64. D. J. Huang, W. Fan, X. C. Li, and Z. Q. Lin, Chin. Opt. Lett. 11, 072301 (2013)

65. D. J. Huang, W. Fan, X. C. Li, and Z. Q. Lin, Chin. Opt. Lett. 10, S21406 (2012).

66. D. J. Huang, W. Fan, X. C. Li, and Z. Q. Lin, Proc. SPIE 8556, 855615 (2012).

67. D. J. Huang, W. Fan, H. Chen, G. Xia, L. L. Pei, X. C. Li, and Z. Q. Lin, Proc. SPIE 10457, 104571P (2017).

68. Y. G. Yao, S. X. Xu, S. X. Meng, and Z. Q. Lin, Acta Opt. Sin. 15, 931 (1995).

69. L. J. Qian, Chin. J. Lasers A 22, 747 (1995).

70. J. M. Auerbach and V. P. Karpenko, Appl. Opt. 33, 3179 (1994).

71. P. Rambo, J. Schwarz, M. Kimmel, and J. L. Porter, High Power Laser Sci. Eng. 4, e32 (2016).

72. M. D. Martinez, J. Crane, L. Hackel, F. Penko, and D. Browning, Proc. SPIE 3267, 234 (1998).

73. J. F. Wang, H. D. Zhu, X. C. Li, and J. Q. Zhu, Chin. J. Lasers 35, 187 (2008).

74. Y. J. Peng, J. F. Wang, X. H. Lu, W. Fan, and X. C. Li, Chin. J. Lasers 41, 0902003 (2014)

75. D. He, S. Kang, L. Zhang, L. Chen, Y. Ding, Q. Yin, and L. Hu, High Power Laser Sci. Eng. 5, e1 (2017). 
76. C. Wang, H. Wei, J. F. Wang, D. J. Huang, and X. C. Li, Chin. Opt. Lett. 15, 011401 (2017).

77. I. Musgrave, W. Shaikh, M. Galimberti, A. Boyle, C. H. Gomez, K. Lancaster, and R. Healthcote, Appl. Opt. 49, 6558 (2010).

78. V. Bagnoud and F. Wagner, High Power Laser Sci. Eng. 4, e39 (2016).
79. T. Huang, X. Pan, P. Zhang, J. Wang, X. Ouyang, and X. Li, Chin. Phys. Lett. 34, 064203 (2017).

80. D. S. Montgomery, in HEDSA Symposium on High Energy Density Laboratory Plasmas (2013), LA-UR-13-285558.

81. J. Zheng, S. W. Wang, and J. Q. Xu, Chin. Phys. Lett 30, 044204 (2013). 